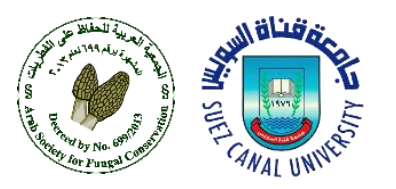

\title{
Checklist of Algerian fungi - Part 5: Dothideomycetes (Ascomycota)
}

\section{Said Amrani ${ }^{1 *}$, Samir Djouadi ${ }^{1}$, Amina Bouherama ${ }^{1}$, Teroj A. Mohamed ${ }^{2}$, Fatma Abo Nouh $^{3}$, Safaa A. Mansour ${ }^{3}$, Sara A. Gezaf ${ }^{4}$, Mohamed A. Abdel Azeem ${ }^{5}$, Hebatallah Abo Nahas ${ }^{6}$, Nourhan E. Abdel-Kader ${ }^{3}$, Maryam T. Elshbrawy ${ }^{3}$, Wassima Lakhdari ${ }^{7}$, Paul Kirk ${ }^{8}$, Ahmed M. Abdel-Azeem ${ }^{3}$}

1* Laboratoire de Biologie et de Physiologie des Organismes, Faculté des Sciences Biologiques, Université des Sciences et de la Technologie Houari Boumediène, BP 32 El Alia, Alger 16111, Algeria.

${ }^{2}$ Dental Basic Sciences Department, College of Dentistry, University of Duhok, Iraq.

${ }^{3}$ Botany and Microbiology Department, Faculty of Science, University of Suez Canal, Ismailia 41522, Egypt.

${ }^{4}$ Botany and Microbiology Department, Faculty of Science, University of Arish, North Sinai, Egypt.

${ }^{5}$ Pharmacognosy Department, Faculty of Pharmacy and Pharmaceutical Industries, University of Sinai, Arish, Egypt.

${ }^{6}$ Zoology Department, Faculty of Science, University of Suez Canal, Ismailia 41522, Egypt.

${ }^{7}$ Department of Plant Protection, Institute of Agronomic Research of Algeria, Algeria.

${ }^{8}$ Biodiversity Informatics \& Spatial Analysis, Royal Botanic Garden Kew, Richmond, London TW9 3AE, United Kingdom.

\section{ARTICLE INFO \\ Article history \\ Accepted 3 May 2021 \\ (C) Amrani et al., 2021 \\ Corresponding Editor: \\ Balbool B \\ Darwish A \\ Keywords \\ Mycobiota \\ Biodiversity \\ Literature and herbaria \\ Catalogues survey \\ Conservation}

Received 20 December 2020

Received revised 28 April 2021

Available online 4 May 2021

\begin{abstract}
Our fifth contribution to the checklist of Algerian fungi is dedicated to Dothideomycetes which comprise a highly diverse range of fungi characterized mainly by asci with double two wall layers (bitunicate asci) and often with fissitunicate dehiscence. By screening all available bibliographic sources and herbaria (fungaria) catalogues it was possible to delineate 562 species belonging to 59 families and 176 genera that occurs in Algeria. Additionally, a list of 10 doubtful records unsupported by literature and/or herbarium (fungarium) material is provided. This checklist is the first comprehensive species list fully dedicated to Algerian Dothideomycetes. Therefore, it should be mentioned here that, although the present study will add some new data to our information concerning Fungi in Algeria, this checklist must be considered as a provisional one always waiting for continuous supplementation.
\end{abstract}

\section{Introduction}

Dothideomycetes is the largest and possibly most phylogenetically diverse class within the phylum, Ascomycota (Kirk et al. 2008, Schoch et al. 2009, Hyde et al. 2013) and is characterized mainly by bitunicate asci

Published by Arab Society for Fungal Conservation

\footnotetext{
* Corresponding author

E-mail address: farida.bettiche@gmail.com (Said Amrani)
}

with fissitunicate dehiscence (Hyde et al. 2013, Kirk et al. 2008).

The class comprises a wide group of fungi that subsist in the majority of the niches where fungi can be 
found. Many species are saprobes, with many asexual states comprising important plant pathogens that cause serious problems to crop plants (Liu et al. 2011, Manamgoda et al. 2012, Ariyawansa et al. 2013a,b, ,). Its members are reported from many plant parts, but less has been reported from wild seed pods and fruits. Dothideomycetes can be seed-borne or colonize fruits and seed pods when they fall to the ground (Jayasiri et al. 2019)

One of the most gaps in knowledge concerning conservation of fungi is the absence of updated check lists for the country. So the information concerning the fungi of Algeria is still incomplete and cannot be fully documented without an updated checklist of all taxa reported for the country.

Taxonomy requires continual updates and checklists are important tools in taxonomy, systematics and conservation (Söderström et al. 2007, 2008; AbdelAzeem and Salem 2013, Nafady et al. 2016, Amrani and Abdel-Azeem 2018a, b, 2019, Amrani et al. 2019, AbdelAzeem et al. 2020).

The goal of the present study is to gather the information available on Dothidiomycetes taxa for Algeria, based on records available in scientific publications and databases since $(18 \varepsilon \cdot)$.

\section{Material and Methods \\ Study area}

For more details on the study area please check our previous work (Amrani and Abdel-Azeem 2018 a,b, Amrani et al. 2019).

\section{Data Collection}

The species listed here were compiled from bibliographical and herbaria online databases sources. A main list of Algerian Dothideomycetes has been developed and the taxa are given in alphabetical sequence of taxa and accepted names are highlighted in bold. The names of authors of fungal taxa are abbreviated according to Kirk and Ansell (1992) and Kirk et al. (2008). Name corrections, authorities reported in this work were checked against the databases of Index fungorum (www.indexfungorum.org).

\section{Abbreviations used}

Herbaria (Fungaria) and Databases that hold specimens or records of the taxa listed in this paper are given between square brackets and abbreviated as:

[BR] Meise Botanic Garden Herbarium (BR)

http://www.botanicalcollections.be/\#/en/home.

[CABI] Descriptions of Fungi and Bacteria

https://www.cabi.org/DFB

[G] Catalogue des Herbiers de Genève

84 http://www.ville-

ge.ch/musinfo/bd/cjb/chg/index.php?lang=en

[GBIF] Global Biodiversity Information Facility https://www.gbif.org/en/.

[ILL] University of Illinois Herbarium

http://mycoportal.org/portal/collections/datasets/datapubli sher.php

[MA] Real Jardín Botánico de Madrid

http://www.rjb.csic.es/jardinbotanico/jardin/index.php?Ca $\mathrm{b}=109$ \&len $=$ en.

[MIN] University of Minnesota herbarium

https://www.bellmuseum.umn.edu/fungi/

[MPU] Herbier de l'Université de Montpellier

https://collections.umontpellier.fr/collections/botanique/he rbier-mpu.

[NCBI] National Center for Biotechnology Information, Nucleotide Database

https://www.ncbi.nlm.nih.gov/nucleotide/

[NY] New York Botanical Garden

http://sciweb.nybg.org/science2/SteereHerbarium.asp

[P] Museum d'Histoire Naturelle de Paris

https://science.mnhn.fr/institution/mnhn/collection/pc//list

[S] Swedish Museum of Natural History

http://herbarium.nrm.se/

[SVH] Sweden's Virtual Herbarium

http://herbarium.emg.umu.se/.

[USDA] U.S. National Fungus Collections

https://nt.ars-grin.gov/fungaldatabases/.

[VKM] Catalog All-Russian Collection of

Microorganisms

http://www.vkm.ru/Catalogue.htm.

\section{Results}

\section{List of accepted taxa}

\section{Aplosporellaceae}

Aplosporella rubicola Maire Bull. Soc. bot. Fr. 53: CLXXXIX (1907) [1906]

syn. Haplosporella rubicola

Ref: Maire (1906), Kedad \& Bouznad (2018).

In fact genus Aplosporella was spelled as Haplosporella by several authors from north Africa from which R. Maire

\section{Arthopyreniaceae}

Arthopyrenia salicis A. Massal. Ric. auton. lich. crost. (Verona): 169 (1852)

Ref: [CABI], [GBIF]. 


\section{Xanthopyreniaceae}

Zwackhiomyces calcariae (Flagey) Hafellner \& Nik. Hoffm. in Hoffmann \& Hafellner, Biblthca Lichenol. 77: 122 (2000)

syn. Arthopyrenia calcariae Flagey, Catal. Lich. Algérie: 113 (1896)

Ref.: Vouaux (1912).

\section{Asterinaceae}

Asterina himantia (Pers.) Sacc., Syll. fung. (Abellini) 1: 761 (1882)

Ref.: [P]

\section{Aulographaceae}

Aulographum hederae Lib. Pl. crypt. Arduenna, fasc. (Liège) 3(nos 201-300): no. 272 (1834)

syn. Aulographum vagum Desm., Annls Sci. Nat., Bot., sér. 2 19: 362 (1843)

Ref.: Durieu (1846), Patouillard (1897b), Kedad \& Bouznad (2018), [GBIF], [P].

\section{Botryosphaeriaceae}

Botryosphaeria corticola A.J.L. Phillips, A. Alves \& J. Luque in Alves, Correia, Luque \& Phillips, Mycologia 96(3): 603 (2004)

Ref.: Belhoucine et al. (2011), Kedad \& Bouznad (2018).

Botryosphaeria dothidea (Moug.) Ces. \& de Not. Comm. Soc. crittog. Ital. 1(fasc. 4): 212 (1863)

Ref.: Kedad \& Bouznad (2018), [NCBI].

Botryosphaeria iberica A.J.L. Phillips, J. Luque \& A. Alves Mycologia 97(2): 524 (2005)

Ref.: Azouaoui-Idjer et al. (2012), Kedad \& Bouznad (2018), [GBIF].

Botryosphaeria malvacearum (Trab.) Weese Sber. Akad. Wiss. Wien, Math.-naturw. Kl., Abt. 1 128: 708 (1919)

syn. Gibberella malvacearum Trab., Revue mycol., Toulouse 9(no. 34): 106 (1887)

Ref.: Roumeguère (1887b), Saccardo (1891), Kedad \& Bouznad (2018), [GBIF].

Botryosphaeria stevensii Shoemaker Can. J. Bot. 42: 1299 (1964)

syn. Diplodia mutila var. major Wollenw. \& Hochapfel, Centbl. Bakt. ParasitKde, Abt. II 12(2): 186 (1941)

Ref.: CMI (1963).
Botryosphaeria trabutiana (Henn.) Theiss. Annls mycol. 14(6): 419 (1917) [1916]

syn. Physalospora trabutiana Henn., Hedwigia 40(Beibl.): (100) (1901)

Ref.: Hennings (1901), Saccardo (1902), Saccardo (1905), Kedad \& Bouznad (2018), [GBIF], [S], [SVH].

Diplodia africana Damm \& Crous Mycologia 99(5): 671 (2008) [2007]

Ref.: [NCBI].

Diplodia agrostidis Sacc. Annls mycol. 3(6): 513 (1906) [1905]

syn. Diplodia ricini Sacc. \& Roum., Revue mycol., Toulouse 3(no. 9): 29 (1881)

Ref.: Saccardo \& Roumeguère (1881), Saccardo (1884), Kedad \& Bouznad (2018).

Diplodia albozonata Durieu \& Mont. in Durieu, Expl. Sci. Alg., Fl. Algér. 1(livr. 15): 573 (1849) [1846-49]

Ref.: Durieu (1846), Montagne (1856), Saccardo (1884), Kedad \& Bouznad (2018), [MPU], [GBIF], [P].

Diplodia arundinacea Durieu \& Mont. in Durieu, Expl. Sci. Alg., Fl. Algér. 1(livr. 15): 574 (1849) [1846-49]

Ref.: Durieu (1846), Montagne (1856), Saccardo (1884), Kedad \& Bouznad (2018), [GBIF], [P].

Diplodia clandestina Durieu \& Mont. in Durieu, Expl. Sci. Alg., Fl. Algér. 1(livr. 15): 575 (1849) [1846-49]

Ref.: Durieu (1846), Montagne (1856), Saccardo (1884), Kedad \& Bouznad (2018), [BR], [GBIF], [P].

Diplodia congesta Lév. Annls Sci. Nat., Bot., sér. 3 5: 290 (1846)

Ref.: [GBIF], [P].

Diplodia consimilis Durieu \& Mont. in Durieu, Expl. Sci. Alg., Fl. Algér. 1(livr. 15): 574 (1849) [1846-49]

Ref.: Durieu (1846), Montagne (1856), Saccardo (1884), Kedad \& Bouznad (2018).

Diplodia corticola A.J.L. Phillips, A. Alves \& J. Luque in Alves, Correia, Luque \& Phillips, Mycologia 96(3): 603 (2004)

Ref.: Smahi et al. (2017), [NCBI].

Diplodia elaeophila Sacc. \& Roum., Revue mycol., Toulouse 3(no. 9): 29 (1881)

Ref.: Saccardo \& Roumeguère (1881), Saccardo (1884), Kedad \& Bouznad (2018).

Diplodia equiseti I.E. Brezhnev Uchen. Zap. Lenin. Univ. Zhdanov 28(biol. sci. 7): 179 (1939) 
Ref.: [GBIF], [P].

Diplodia euonymi Westend [as 'evonymi'], in Kickx, Fl. Crypt. Flandres (Paris) 1: 395 (1867)

Ref.: [MPU], [GBIF].

Diplodia fissa Durieu \& Mont. in Durieu, Expl. Sci Alg., Fl. Algér. 1 livr. 15): 572 (1849) [1846-49]

Ref.: Durieu (1846), Montagne (1856), Saccardo (1884), Kedad \& Bouznad (2018), [GBIF], [P].

Diplodia herbarum (Corda) Lév. Annls Sci. Nat., Bot., sér. 3 5: 292 (1846)

Ref.: Saccardo \& Roumeguère (1881) 100, Kedad \& Bouznad (2018), [GBIF], [P].

Diplodia heteroclita Durieu \& Mont. in Durieu, Expl. Sci. Alg., Fl. Algér. 1(livr. 15): 576 (1849) [1846-49]

Ref.: Durieu (1846), Montagne (1856), Saccardo (1882a), Kedad \& Bouznad (2018), [GBIF], [P].

Diplodia microspora f. araucariae Sacc. \& Roum. Reliq. Libert, Ser. 4: no. 140 (1884)

Ref.: Saccardo \& Roumeguère (1881), Saccardo \& Roumeguère (1881), Saccardo (1884), Kedad \& Bouznad (2018).

Diplodia olivarum A.J.L. Phillips, Frisullo \& Lazzizera Fungal Diversity 31: 67 (2008)

Ref.: [NCBI].

Diplodia philodendri Tassi Bulletin Labor. Orto Bot. de R. Univ. Siena 2: 155 (1899)

Ref.: [P].

Diplodia quercivora Linald. \& A.J.L. Phillips Mycologia 105(5): 1269 (2013)

Ref.: Linaldeddu et al. (2013), Smahi et al. (2017).

Diplodia rehmii Bäumler Hedwigia 24: 75 (1885)

Ref.: Larignon (2016), [MPU].

Diplodia seriata de Not. Mém. R. Accad. Sci. Torino, Ser. 2 7: 26 (1845)

Ref.: Kedad \& Bouznad (2018), [NCBI].

Diplodia suberina Durieu \& Mont. in Durieu, Expl. Sci. Alg., Fl. Algér. 1(livr. 15): 572 (1849) [1846-49]

Ref.: Durieu (1846), Montagne (1856), Saccardo (1884), Kedad \& Bouznad (2018), [GBIF], [P].

Diplodia uredinicola Desm. Annls Sci. Nat., Bot., sér. 3 14: 114 (1850)

Ref.: Roumeguère (1887c).

Dothiorella iberica A.J.L. Phillips, J. Luque \& A. Alves Mycologia 97(2): 524 (2005)
Dothiorella oxycedri Maire Bull. Soc. bot. Fr. 53: CLXXXVIII (1907) [1906]

Ref.: Maire (1906), Kedad \& Bouznad (2018).

Dothiorella sarmentorum (Fr.) A.J.L. Phillips, A. Alves \& J. Luqu Mycologia 97(2): 522 (2005)

syn. Sphaeria sarmentorum Fr., K. svenska VetenskAkad. Handl., ser. 3 40: 107 (1819)

Ref.: [GBIF], [P].

Fusicoccum asparagi S. Ahmad Sydowia 5(3-6): 390 (1951)

Ref.: [MPU].

Fusicoccum sardoa (Pass.) Vanev in van der Aa \& Vanev, A Revision of the Species Described in Phyllosticta (Utrecht): 414 (2002)

syn. Phyllosticta sardoa Pass., Atti Accad. naz. Lincei Memorie, Sér. 2 4: 8 (1890)

Ref.: Stevenson (1926).

Lasiodiplodia exigua Linald., Deidda \& A.J.L. Phillips in Linaldeddu, Deidda, Scanu, Franceschini, Serra, Berraf-Tebbal, Zouaoui, Boutiti, Jama \& Phillips, Fungal Diversity 71: 207 (2014)

Ref.: Linaldeddu et al. (2014), Smahi et al. (2017), [NCBI].

Lasiodiplodia frezaliana Faurel \& Schotter Bull. Soc. Hist. nat. Afr. N. 47: 121 (1956)

Ref.: Faurel \& Schoter (1956), CMI (1963).

Lasiodiplodia mediterranea Linald., Deidda \& A.J.L. Phillips in Linaldeddu, Deidda, Scanu, Franceschini, Serra, Berraf-Tebbal, Zouaoui, Boutiti, Jama \& Phillips, Fungal Diversity 71: 207 (2014) [2015]

Ref.: Linaldeddu et al. (2014), Kedad \& Bouznad (2018), [NCBI].

Lasiodiplodia theobromae (Pat.) Griffon \& Maubl. Bull. Soc. mycol. Fr. 25: 57 (1909)

syn. Sphaeria glandicola Schwein., Trans. Am. phil. Soc., New Series 4(2): 214 (1832) [1834]

Ref.: [GBIF], [P].

Macrophoma collabens Berl. \& Voglino, Atti Soc. Veneto-Trent. Sci. Nat. 10(1): 180 (1886)

syn. Phoma collabens Durieu \& Mont. Expl. Sci. Alg., Fl. Algér. 2(livr. 20): 607 (1855)

Ref.: Montagne (1856), Saccardo (1884).

Macrophoma araliae Sacc. \& Berl. Revue mycol., Toulouse 8(no. 29): 35 (1886)

Ref.: Saccardo \& Berlese (1886a), Saccardo (1886a), Kedad \& Bouznad (2018), [MPU], [GBIF]. 
Macrophoma crozalsii Maire Annls mycol. 11(4): 354 (1913)

Ref.: Maire (1913), Saccardo (1931), [MPU], [GBIF].

Macrophoma flaccida (Viala \& Ravaz) Cavara Atti Ist. bot. R. Univ. Pavia, 2 Sér. 1: 317 (1888)

Ref.: Maire \& Werner (1937), Kedad \& Bouznad (2018).

Macrophoma haloxyli Henn. Annuaire Conser. et Jard. bot. Genève 7-8: 243 (1904)

Ref.: Saccardo (1906), Hennings (1902), Kedad \& Bouznad (2018).

Macrophoma hochreutineri Henn. Annuaire Conser. et Jard. bot. Genève 7-8: 243 (1904)

Ref.: Hennings (1902), Kedad \& Bouznad (2018).

Macrophoma macrochloae Trab. Fungi Selecti Galliaei Exs., Cent. 39: no. 4079 (1886)

Ref.: Roumeguère (1887b), Durieu (1846), Kedad \& Bouznad (2018), [GBIF], [P].

Macrophoma malcomiae (Sacc.) Berl. \& Voglino Atti Soc. Veneto-Trent. Sci. Nat. 10(1): 177 (1886)

syn. Phoma malcomiae Sacc., Revue mycol., Toulouse 7(no. 26): 158 (1885)

Ref.: Roumeguère (1886), Saccardo (1886a), Saccardo (1892), Kedad \& Bouznad (2018), [BR], [S], [SVH], [GBIF].

Macrophoma mantegazziana (Penz.) Berl. \& Voglino Atti Soc. Veneto-Trent. Sci. Nat. 10(1): 193 (1886)

Ref.: Saccardo (1886a), Saccardo (1892), Kedad \& Bouznad (2018).

Macrophoma sophorae I. Miyake Bot. Mag., Tokyo 26: 59 (1912)

Ref.: [MPU].

Microdiplodia depazeoides (Durieu \& Mont.) Zambett. Bull. trimest. Soc. mycol. Fr. 70(3): 248 (1955) [1954]

Syn. Ascochytella depazeoides (Durieu \& Mont.) Tassi, Bulletin Labor. Orto Bot. de R. Univ. Siena 5: 27 (1902), Diplodia depazeoides Durieu \& Mont., in Durieu, Expl. Sci. Alg., Fl. Algér. 1(livr. 15): 575 (1849) [1846-49]

Ref.: Durieu (1846), Montagne (1856), Saccardo (1884), Stevenson (1926), Kedad \& Bouznad (2018).

Microdiplodia gayi (G. Boyer \& Jacz.) Allesch. Rabenh. Krypt.-Fl., Edn 2 (Leipzig) 1(7): 94 (1901) [1903]

Ref.: [MPU].
Microdiplodia microsporella Allesch. Rabenh. Krypt.Fl., Edn 2 (Leipzig) 1(7): 79 (1901) [1903]

Ref.: Maire \& Werner (1937), Kedad \& Bouznad (2018).

Microdiplodia passeriniana (Thüm.) Allesch. Rabenh. Krypt.-Fl., Edn 2 (Leipzig) 1(7): 91 (1901) [1903]

syn. Diplodia passeriniana Thüm., Nuovo G. bot. ital. 8(3): 251 (1876)

Ref.: Roumeguère (1882), [BR], [GBIF].

Microdiplodia perpusilla (Desm.) Allesch. Rabenh. Krypt.-Fl., Edn 2 (Leipzig) 1(7): 86 (1901) [1903]

Syn. Diplodia perpusilla Desm., Annls Sci. Nat., Bot., sér. 3 6: 68 (1846)

Ref.: Durieu (1846), Kedad \& Bouznad (2018), [GBIF], [P].

Neodeightonia phoenicum A.J.L. Phillips \& Crous. in Phillips, Alves, Pennycook, Johnston, Ramaley, Akulov \& Crous, Persoonia 21: 43 (2008)

Ref.: Kedad \& Bouznad (2018).

Neofusicoccum algeriense Berr.-Tebb. \& A.J.L. Phillips. in Berraf-Tebbal, Guerreiro \& Phillips, Fungal Diversity 53: 423 (2014)

Ref.: Berraf-Tebbal et al. (2014), Larignon (2016), Kedad \& Bouznad (2018).

Neofusicoccum australe (Slippers, Crous \& M.J. Wingf.) Crous, Slippers \& A.J.L. Phillips in Crous, Slippers, Wingfield, Rheeder, Marasas, Phillips, Alves, Burgess, Barber \& Groenewald, Stud. Mycol. 55: 248 (2006)

Ref.: Larignon (2016), Kedad \& Bouznad (2018), [NCBI].

Neofusicoccum cryptoaustrale Pavlic, Maleme, Slippers \& M.J. Wingf. in Crous et al., Persoonia 31: 271 (2013)

Ref.: [NCBI].

Neofusicoccum luteum (Pennycook \& Samuels) Crous, Slippers \& A.J.L. Phillips. in Crous, Slippers, Wingfield, Rheeder, Marasas, Phillips, Alves, Burgess, Barber \& Groenewald, Stud. Mycol. 55: 248 (2006)

Ref.: [NCBI].

Neofusicoccum mediterraneum Crous, M.J. Wingf. \& A.J.L. Phillips. Fungal Planet, no. 11-21: 19: [2] (2007)

Ref.: Berraf-Tebbal et al. (2014), Larignon (2016), Kedad \& Bouznad (2018), [NCBI].

Neofusicoccum parvum (Pennycook \& Samuels) Crous, Slippers \& A.J.L. Phillips. in Crous, Slippers, 
Wingfield, Rheeder, Marasas, Phillips, Alves, Burgess, Barber \& Groenewald, Stud. Mycol. 55: 248 (2006)

Ref.: Berraf-Tebbal et al. (2014), Larignon (2016), Kedad \& Bouznad (2018), [NCBI].

Neofusicoccum ribis (Slippers, Crous \& M.J. Wingf.) Crous, Slippers \& A.J.L. Phillips. in Crous, Slippers, Wingfield, Rheeder, Marasas, Phillips, Alves, Burgess, Barber \& Groenewald, Stud. Mycol. 55: 249 (2006)

syn. Dothiorella gregaria Sacc., Michelia 2(no. 7): 343 (1881)

Ref.: Roumeguère (1887b), Larignon (2016).

Neoscytalidium dimidiatum (Penz.) Crous \& Slippers. in Crous, Slippers, Wingfield, Rheeder, Marasas, Phillips, Alves, Burgess, Barber \& Groenewald, Stud. Mycol. 55: 244 (2006)

syn. Hendersonula toruloidea Nattrass, Trans. Br. mycol. Soc. 18(3): 197 (1933)

Ref.: Mariat et al. (1978), [GBIF].

Sphaeropsis hedericola (Speg.) Sacc. Syll. fung. (Abellini) 3: 295 (1884)

Ref.: [MPU], [GBIF].

Sphaeropsis sapinea (Fr.) Dyko \& B. Sutton. in Sutton, The Coelomycetes (Kew): 120 (1980)

syn. Diplodia sapinea (Fr.) Fuckel, Jb. nassau. Ver. Naturk. 23-24: 393 (1870) [1869-70]

Ref.: Smahi et al. (2017).

\section{Camarosporidiellaceae}

Camarosporidiella elongata (Fr.) Wanas., Wijayaw. \& K.D. Hyde, in Wanasinghe, Hyde, Crous, Wijayawardene, Jeewon, Jones, Bhat, Phillips, Groenewald, Dayarathne, Phukhamsakda, Thambugala, Bulgakov, Camporesi, Gafforov, Mortimer \& Karunarathna, Stud. Mycol. 87: 230 (2017)

syn. Camarosporium elongatum (Fr.) Wijayaw. \& K.D. Hyde [as 'elongata'], in Wijayawardene, Hyde, Bhat, Camporesi, Schumacher, Chethana, Wikee, Bahkali \& Wang, Cryptog. Mycol. 35(2): 183 (2014), Cucurbitaria elongata (Fr.) Grev., Scott. crypt. fl. (Edinburgh) 4: 195 (1825), Sphaeria elongata Fr., Observ. mycol. (Havniae) 1: 175 (1815)

Ref.: Durieu (1846), Kedad \& Bouznad (2018), [GBIF], [P].

\section{Capnodiaceae}

Antennariella elaeophila (Mont.) Bat. \& Cif. - Quad. Lab. crittogam., Pavia 31: 27 (1963)

syn. Antennaria elaeophila
Ref.: [MIN].

Capnodium lanosum Cooke. Grevillea 8(no. 47): 96 (1880)

Ref.: [PlutoF], [GBIF].

Morfea helianthemi (Maire) Bat. \& Cif. Saccardoa 2: 146 (1963)

syn. Limacinia helianthemi Maire, Annls mycol. 4(4): 331 (1906)

Ref.: Maire (1906), Saccardo (1913), Kedad \& Bouznad (2018).

Polychaetella araucariae (Thüm.) Speg. Physis, Rev. Soc. Arg. Cienc. Nat. 4(no. 17): 295 (1918)

syn. Capnodium araucariae Thüm., Mycoth. Univ., cent. 4: no. 311 (1878)

Ref.: Saccardo \& Berlese (1886a), Kedad \& Bouznad (2018).

\section{Cladosporiaceae}

Cladosporium algeriense (Montpell.) Vuill. Encyclop. Mycol. (Paris) 2: 283 (1931)

syn. Hormodendrum algeriense Montpell. [as 'algeriensis'], Ann. Dermatol. Syph., sér. 5 8(6): 626 (1927)

Ref.: Petrak (1920-39), Petrak (1929).

Cladosporium cladosporioides (Fresen.) G.A. de Vries. Contrib. Knowledge of the Genus Cladosporium Link ex Fries: 57 (1952)

Ref.: Locquin-Linard (1988), Kahoul et al. (2014), Bensaci et al. (2015), Rebbouh (2016), Abdelaziz et al. (2018), [GBIF].

Cladosporium cucumerinum Ellis \& Arthur. Bull. Indiana Agric. Stat. 19: 9 (1889)

Ref.: Kedad \& Bouznad (2018).

Cladosporium delicatulum Cooke. Grevillea 5(no. 33): 17 (1876)

Ref.: [NCBI].

Cladosporium fumago Link. in Willdenow, Sp. pl., Edn 4 6(1): 40 (1824)

Ref.: Durieu (1846) Kedad \& Bouznad (2018).

Cladosporium herbarum (Pers.) Link. Mag. Gesell. naturf. Freunde, Berlin 8: 37 (1816) [1815]

Ref.: Durieu (1846), Saccardo \& Roumeguère (1881), Trabut (1891), Ladjal (2012), Chliyeh et al. (2014), Kedad \& Bouznad (2018), [BR], [GBIF], [P].

Cladosporium iridis (Fautrey \& Roum.) G.A. de Vries. Contrib. Knowledge of the Genus Cladosporium Link ex Fries: 49 (1952) 
syn. Brachysporium gracile (Wallr.) Sacc., Syll. fung. (Abellini) 4: 430 (1886), Davidiella macrospora (Kleb.) Crous \& U. Braun, in Braun, Crous, Dugan \& de Hoog, Mycol. Progr. 2(1): 10 (2003)

Ref.: Maire (1915c), Kedad \& Bouznad (2018), [S], [SVH], Biodiv. Atlas Swed., [GBIF].

Cladosporium macrocarpum Preuss. in Sturm, Deutschl. Fl., 3 Abt. (Pilze Deutschl.) [6](25-26): 27 (1848)

syn. Davidiella macrocarpa Crous, K. Schub. \& U. Braun, in Schubert, Groenewald, Braun, Dijksterhuis, Starink, Hill, Zalar, de Hoog \& Crous, Stud. Mycol. 58: 129 (2007)

Ref.: Saccardo \& Roumeguère (1881), Kachour (2004), Kedad \& Bouznad (2018).

Cladosporium microporum Rabenh. in Sturm, Deutschl. Fl., 3 Abt. (Pilze Deutschl.) [6](25-26): 27 (1848)

Ref.: [BR], [GBIF], [GBIF].

Cladosporium nervisequum Mont. Annls Sci. Nat., Bot., sér. 4 8: 298 (1857)

Ref.: Montagne (1857).

Cladosporium ramotenellum K. Schub., Zalar, Crous \& U. Braun. in Schubert, Groenewald, Braun, Dijksterhuis, Starink, Hill, Zalar, de Hoog \& Crous, Stud. Mycol. 58: 137 (2007)

Ref.: Chamekh et al. (2019).

Cladosporium sphaerospermum Penz. in Saccardo, Michelia 2(no. 8): 473 (1882)

Ref.: Kachour (2004), [GBIF].

Cladosporium typharum Desm. Pl. crypt. exsicc. 1: no. 304 (1834)

syn. Cladosporium typharum f. minor Brunaud, Bull. Soc. bot. Fr. 36: 340 (1889)

Ref.: Saccardo \& Roumeguère (1881), Maire (1906), Kedad \& Bouznad (2018).

Polyrhizium leptophyei (Giard) Giard [as 'leptophyae'], Bull. Sci. France Belgique 20: 217 (1889)

Ref.: Trabut (1891).

\section{Coniothyriaceae}

Coniothyrium borbonicum Thüm. Inst. Coimbra: no. 339 (1879)

Ref.: [BR], [GBIF], [USDA]

Coniothyrium concentricum (Desm.) Sacc. Michelia 1(no. 2): 204 (1878) syn. Coniothyrium concentricum var. agaves Sacc., Syll. fung. (Abellini) 3: 317 (1884)

Ref.: [P].

Coniothyrium palmarum Corda. Icon. fung. (Prague) 4: 38 (1840)

Ref.: Saccardo \& Berlese (1886a), Kedad \& Bouznad (2018), [GBIF], [P].

\section{Cucurbitariaceae}

Cucurbitaria asparagi Maire. Bull. trimest. Soc. mycol. Fr. 40(4): 316 (1926) [1924]

Ref.: Kedad \& Bouznad (2018), [MPU], [GBIF].

Cucurbitaria dulcamarae (Kunze \& J.C. Schmidt) Fr. Summa veg. Scand., Sectio Post. (Stockholm): 391 (1849)

syn. Sphaeria dulcamara Kunze \& J.C. Schmidt, Mykologische Hefte (Leipzig) 1: 62 (1817)

Ref.: Durieu (1846), Kedad \& Bouznad (2018), [P].

Cucurbitaria spartii (Nees ex Fr.) Ces. \& de Not. Comm. Soc. crittog. Ital. 1(fasc. 4): 214 (1863)

syn. Sphaeria spartii Nees ex Fr., in Schmidt \& Kunze, Deutschl. Schwämme, Achte Lieferung: 2 (1818)

Ref.: Durieu (1846), Kedad \& Bouznad (2018).

\section{Davidiellaceae}

Davidiella ammophilae (Durieu \& Mont.) Aptroot. CBS Diversity Ser. (Utrecht) 5: 33 (2006)

syn. Asterina ammophilae Durieu \& Mont., in Durieu, Expl. Sci. Alg., Fl. Algér. 1(livr. 14): 548 (1848) [1846-49]

Ref.: Durieu (1846), Montagne (1856), Saccardo (1882b), Kedad \& Bouznad (2018).

Dichocladosporium chlorocephalum (Fresen.) Trail. in Schubert, Braun, Groenewald \& Crous, Stud. Mycol. 58: 96 (2007)

syn. Haplographium chlorocephalum (Fresen.) Grove, Hardwick's Science Gossip, (London) 21: 198 (1885)

Periconia ellipsospora Penz. \& Sacc., Atti Inst. Veneto Sci. lett., ed Arti, Sér. 6 2: 596 (1884)

Ref.: Killian \& Fehér (1935).

\section{Delitschiaceae}

Delitschia auerswaldii Fuckel. Jb. nassau. Ver. Naturk. 23-24: 241 (1870) [1869-70]

Ref.: Faurel \& Schotter (1964ba).

Delitschia marchalii Berl. \& Voglino.in Saccardo, Syll. fung., Addit. I-IV (Abellini): 127 (1886) 
Ref.: Locquin-Linard (1988).

Delitschia patagonica Speg. Boln Acad. nac. Cienc. Córdoba 11(1): 44 (1887)

Ref.: Locquin-Linard (1988).

\section{Diademaceae}

Clathrospora diplospora (Ellis \& Everh.) Sacc. \& Traverso. Syll. fung. (Abellini) 19: 308 (1910)

Ref.: Lacoste (1957).

Clathrospora permunda (Cooke) Berl. Nuovo G. bot. ital. 20(2): 194 (1888)

Ref.: Berlese (1892-1905).

\section{Diaporthaceae}

Phomopsis caulographa (Durieu \& Mont.) Grove, Bull. Misc. Inf., Kew(no. 2): 54 (1917)

syn. Phoma caulographa Durieu \& Mont., Expl. Sci. Alg., Fl. Algér. 2(livr. 20): 606 (1855)

Ref.: Durieu (1846), Saccardo (1884).

Phomopsis sapindi (Pat.) Sacc., G. bot. ital., n.s. 22(1): 49 (1915)

syn. Phoma sapindi Pat., Bull. Soc. mycol. Fr. 19(3): 259 (1903)

Ref.: Saccardo (1906), Patouillard (1903), Pfister (1977), Kedad \& Bouznad (2018).

\section{Didymellaceae}

Ascochyta alceina Lambotte \& Fautrey. Bull. Soc. mycol. Fr. 15: [153] (1899)

Ref.: Kedad \& Bouznad (2018).

Ascochyta avenae (Petr.) R. Sprague \& Aar.G. Johnson. Mycologia 40(3): 297 (1948)

Ref.: [CABI].

Ascochyta boehmeriae Maire. Bull. Soc. Hist. nat. Afr. N. 36(3): 42 (1945)

Ref.: Maire (1945), CMI (1954), Kedad \& Bouznad (2018), [G], [MPU], [GBIF].

Ascochyta cynarae Maffei. Atti Ist. bot. R. Univ. Pavia, 2 Sér. 12: 10 (1909)

Ref.: Kedad \& Bouznad (2018).

Ascochyta equiseti (Desm.) Grove. J. Bot., Lond. 56: 315 (1918)

syn. Phoma equiseti Desm., Pl. Crypt. Nord France, Edn 12 : no. 183 (1846)

Ref.: [P].

Ascochyta kentiae Maubl. Bull. Soc. mycol. Fr. 19(3): 293 (1903)
Ref.: Saccardo (1906), Maublanc (1903), Stevenson (1926), Mouchacca \& Saint-Jore (2000), Mouchacca (2000), Kedad \& Bouznad (2018).

Ascochyta medicaginicola Qian Chen \& L. Cai, in Chen, Jiang, Zhang, Cai \& Crous, Stud. Mycol. 82: 187 (2015)

syn. Phoma medicaginis Malbr. \& Roum., in Roumeguère, Fungi Selecti Galliaei Exs., Cent. 37: no. 3675 (1886)

Ref.: Kedad \& Bouznad (2018).

Boeremia exigua (Desm.) Aveskamp, Gruyter \& Verkley. in Aveskamp, Gruyter, Woudenberg, Verkley \& Crous, Stud. Mycol. 65: 37 (2010)

Ref.: Belhoucine et al. (2011), Kedad \& Bouznad (2018), [MIN].

Boeremia foveata (Foister) Aveskamp, Gruyter \& Verkley. in Aveskamp, Gruyter, Woudenberg, Verkley \& Crous, Stud. Mycol. 65: 40 (2010)

Ref.: Kedad \& Bouznad (2018).

Boeremia hedericola (Durieu \& Mont.) Aveskamp, Gruyter \& Verkley, in Aveskamp, Gruyter, Woudenberg, Verkley \& Crous, Stud. Mycol. 65: 40 (2010)

syn. Phyllosticta hedericola Durieu \& Mont., in Montagne, Syll. gen. sp. crypt. (Paris): 279 (1856)

Ref.: Montagne (1856), Saccardo (1884), Stevenson (1926), Kedad \& Bouznad (2018).

Didymella anserina (Marchal) Qian Chen \& L. Cai. in Chen, Jiang, Zhang, Cai \& Crous, Stud. Mycol. 82: 173 (2015)

syn. Phoma anserina Marchal, Champ. copr. Belg. 6: 11 (1891)

Ref.: Mouchacca (2000).

Didymella calidophila (Aveskamp, Gruyter \& Verkley) Q. Chen \& L. Cai. in Chen, Jiang, Zhang, Cai \& Crous, Stud. Mycol. 82: 174 (2015)

syn. Sphaeronaema sahariense Faurel \& Schotter, Revue Mycol., Paris 30(3): 156 (1965) Ref.: Faurel \& Schotter (1965), Locquin-Linard (1988).

Didymella fabae G.J. Jellis \& Punith. Pl. Path. 40(1): 151 (1991)

Ref.: Kedad \& Bouznad (2018).

Didymella glomerata (Corda) Qian Chen \& L. Cai. in Chen, Jiang, Zhang, Cai \& Crous, Stud. Mycol. 82: 176 (2015)

syn. Phoma glomerata (Corda) Wollenw. \& Hochapfel, Z. ParasitKde 3(5): 592 (1936) 
Ref.: Locquin-Linard (1988), Belhoucine et al. (2011), Kedad \& Bouznad (2018), [P].

Didymella hyphenis (Cooke) Sacc. Michelia 2(no. 7): 316 (1881)

syn. Sphaeria hyphenis Cooke, Handb. Brit. Fungi 2: 895 (1871)

Ref.: Debeaux et al. (1880), Saccardo (1882b), Kedad \& Bouznad (2018), [NY], [GBIF].

Didymella lentis W.J. Kaiser, B.C. Wang \& J.D. Rogers. Pl. Dis. 81(7): 815 (1997)

Ref.: Kedad \& Bouznad (2018).

Didymella pinodella (L.K. Jones) Qian Chen \& L. Cai. in Chen, Jiang, Zhang, Cai \& Crous, Stud. Mycol. 82: 178 (2015)

Ref.: Kedad \& Bouznad (2018).

Didymella pinodes (Berk. \& A. Bloxam) Petr Annls mycol. 22(1/2): 16 (1924)

syn. Mycosphaerella pinodes (Berk. \& A. Bloxam) Vestergr., Annls mycol. 10(6): 581 (1912)

Ref.: Setti et al. (2009), Kedad \& Bouznad (2018).

Didymella pisi Chilvers, J.D. Rogers \& Peever. Mycol. Res. 113(3): 396 (2009)

syn. Ascochyta pisi Lib., Pl. crypt. Arduenna, fasc. (Liège) 1(nos 1-100): no. 59 (1830)

Ref.: Maire (1917b), Maire (1919), Kedad \& Bouznad (2018), Tadja (2018), [G], [MA], [MPU], [GBIF], [USDA].

Didymella rabiei Kovatsch. ex Arx, in Müller \& von Arx, Beitr. Kryptfl. Schweiz 11(no. 2): 364 (1962)

Ref.: Maire \& Werner (1937), Kedad \& Bouznad (2018).

Didymella viciae Bouznad [anamorph of Septoria viciae Lib.]. in Bouznad, Roquebert \& Benelmouffok, Mycotaxon 56: 445 (1995)

Ref.: Bouznad et al. (1995), Kedad \& Bouznad (2018), [GBIF].

Dothiora oleae (DC.) Crous, in Crous \& Groenewald, Fungal Biology 120(11): 1409 (2016)

Syn. Phoma oleae (DC.) Sacc., Michelia 2(no. 6): 91 (1880)

Ref.: Patouillard (1897b), Kedad \& Bouznad (2018).

Epicoccum andropogonis (Ces.) Schol-Schwarz. Trans. Br. mycol. Soc. 42(2): 171 (1959)

syn. Cerebella andropogonis Ces., in Rabenhorst, Bot. Ztg. 9: 669 (1851)
Ref.: Patouillard (1903), Maire \& Werner (1937), Kedad \& Bouznad (2018).

Epicoccum nigrum Link, Mag. Gesell. naturf. Freunde, Berlin 7: 32 (1816) [1815]

syn. Epicoccum vulgare Corda, Icon. fung. (Prague) 1: 5 (1837)

Ref.: Durieu (1846), Nicot (1955), Dorléans \& Emmanouilidis (1972), Bouharati et al. (2008), Harzallah et al. (2009), Kedad \& Bouznad (2018).

Juxtiphoma eupyrena (Sacc.) Valenz.-Lopez, Crous, Stchigel, Guarro \& Cano. in Valenzuela-Lopez, CanoLira, Guarro, Sutton, Wiederhold, Crous \& Stchigel, Stud. Mycol. 90: 40 (2017)

syn. Phoma eupyrena Sacc., Michelia 1(no. 5): 525 (1879)

Ref.: Kahoul et al. (2014).

Paraboeremia putaminum (Speg.) Qian Chen \& L. Cai. in Chen, Jiang, Zhang, Cai \& Crous, Stud. Mycol. 82: 184 (2015)

syn. Phoma putaminum Speg., Atti Soc. Crittogam. Ital. 3(1): 66 (1881)

Ref.: Chafai (1996).

Paratrichaegum stercorarium Faurel \& Schotter. Revue Mycol., Paris 30(5): 348 (1966) [1965]

Ref.: CMI (1968).

Peyronellaea stipae Lacoste. Revue Mycol., Paris 22(Suppl. Colon): 14 (1957)

Ref.: Lacoste (1957), CMI (1963), [P].

Phoma agaves Durieu \& Mont. Expl. Sci. Alg., Fl. Algér. 2(livr. 20): 605 (1855)

Ref.: Montagne (1856).

Phoma alleizetteana Gonz. Frag. Brotéria, sér. bot. 21: 119 (1924)

Ref.: [MA], [GBIF].

Phoma ammophilae Durieu \& Mont. in Durieu, Expl. Sci. Alg., Fl. Algér. 1(livr. 15): 564 (1849) [1846-49]

Ref.: Roumeguère (1886), [BR], [GBIF].

Phoma arbuti Maire. Results of the investigation of the beet eelworm Heterodera Schachtii Schmidt, conduced at the labor 1: 126 (1916)

Ref.: Saccardo (1931), [MPU], [GBIF].

Phoma bolleana Thüm. Boll. Soc. Adriatica Sci. Nat. Triests 2: 453 (1877)

Ref.: Roumeguère (1888b), [BR], [GBIF]. 
Phoma calligoni Murashk. Trans. Agric. Forest. omsk. 9: 6 (1928)

Ref.: [MA], [GBIF].

Phoma cookei Pirotta. Riv. Vitic. anolog. Conegl. 3(no. 23): 55 (1879)

Ref.: [USDA]

Phoma crucianellae (Durieu \& Mont.) Sacc. Syll. fung. (Abellini) 3: 138 (1884)

Ref.: Saccardo (1884), Kedad \& Bouznad (2018).

Phoma debeauxiana Sacc. Syll. fung. (Abellini) 3: 160 (1884)

Ref.: Saccardo (1884), Kedad \& Bouznad (2018), [S], [SVH], [GBIF].

Phoma deserticola Faurel \& Schotter. Revue Mycol., Paris 30(3): 154 (1965)

Ref.: Faurel \& Schotter (1965), Locquin-Linard (1988).

Phoma durandoi Maire. Bull. Soc. Hist. nat. Afr. N. 36(3): 41 (1945)

Ref.: CMI (1954), Kedad \& Bouznad (2018).

Phoma hedysari Durieu \& Mont. Expl. Sci. Alg., Fl. Algér. 2(livr. 20): 608 (1855)

Ref.: Montagne (1856), Saccardo (1884), Kedad \& Bouznad (2018).

Phoma herbarum Westend. Bull. Acad. R. Sci. Belg., Cl. Sci. 19(no. 3): 118 (1852)

syn. Phoma tetragoniae (Sacc. \& Berl.) Mussat, in Saccardo, Syll. fung. (Abellini) 15: 286 (1901)

Ref.: Saccardo \& Berlese (1886a), Saccardo (1886a), Saccardo (1892), Dubost (1966), Locquin-Linard (1988), Bekhouche et al. (1994), Kedad \& Bouznad (2018).

Phoma hoggariana Faurel \& Schotter. Revue Mycol., Paris 30(3): 153 (1965)

Ref.: Faurel \& Schotter (1965).

Phoma ilicis Desm. Pl. Crypt. Nord France, Edn 1: no. 1290 (1843)

Ref.: Kedad \& Bouznad (2018), [BR], [GBIF].

Phoma lentiscina Maire. Bull. Soc. Hist. nat. Afr. N. 8(7): 180 (1917)

Ref.: Maire (1917a), Kedad \& Bouznad (2018), [MPU], [GBIF].

Phoma microspora Sacc. Revue mycol., Toulouse 7(no. 26): 158 (1885)
Ref.: Roumeguère (1885), Saccardo \& Roumeguère (1885), Saccardo (1886a), Saccardo (1892), Kedad \& Bouznad (2018), [BR], [S], [SVH], [GBIF].

Phoma musarum Cooke. Grevillea 12(no. 61): 22 (1883)

Ref.: Maire (1917b), Kedad \& Bouznad (2018), [MA], [MPU], [GBIF], [USDA]

Phoma myriocarpa Durieu \& Mont. in Durieu, Expl. Sci. Alg., Fl. Algér. 2(livr. 20): 601 (1855)

Ref.: Montagne (1856), Saccardo (1884), Kedad \& Bouznad (2018).

Phoma nivellei Maire. Bull. Soc. Hist. nat. Afr. N. 22: 22 (1931)

Ref.: Maire (1933), Kedad \& Bouznad (2018), [MPU], [GBIF].

Phoma radicicola McAlpine, Fungus Diseases of stone-fruit trees in Australia: 126 (1902)

Ref.: Saccardo (1906), Kedad \& Bouznad (2018).

Phoma rhabdosporica J.V. Almeida \& Sousa da Câmara. Bolm Soc. broteriana, Coimbra, sér. 1 24: 36 (1909)

Ref.: [MPU].

Phoma rosarum Durieu \& Mont. in Durieu, Expl. Sci. Alg., Fl. Algér. 2(livr. 20): 601 (1855)

Ref.: Montagne (1856), Saccardo (1884), Kedad \& Bouznad (2018).

Phoma sahariensis Faurel \& Schotter. Revue Mycol., Paris 30(3): 155 (1965)

Ref.: Faurel \& Schotter (1965), Locquin-Linard (1988).

Phoma solieri (Mont.) Sacc. Michelia 1(no. 5): 525 (1879)

syn. Macrophoma solieri (Mont.) Berl. \& Voglino, Atti Soc. Veneto-Trent. Sci. Nat. 10(1): 190 (1886)

Ref.: Maire (1906), Kedad \& Bouznad (2018), [MPU], [GBIF].

Phoma sordida Durieu \& Mont. Expl. Sci. Alg., Fl. Algér. 2(livr. 20): 607 (1855)

Ref.: Montagne (1856), Saccardo (1884), Kedad \& Bouznad (2018).

Phoma stigmea Durieu \& Mont. in Durieu, Expl. Sci. Alg., Fl. Algér. 2(livr. 20): 606 (1855)

Ref.: Montagne (1856), Saccardo (1882a), Saccardo (1884), Stevenson (1926), Kedad \& Bouznad (2018).

Phoma subconfluens Mont. \& Durieu in Durieu, Expl. Sci. Alg., Fl. Algér. 2(livr. 20): 601 (1855) 
Ref.: Montagne (1856), Saccardo (1884), Kedad \& Bouznad (2018).

Phoma tefedestica Faurel \& Schotter. Revue Mycol., Paris 30: 155 (1965)

Ref.: Faurel \& Schotter (1965), Locquin-Linard (1988).

Phoma telmatejae Brunaud. Act. Soc. linn. Bordeaux 44: 36 (1890)

Ref.: [MPU], [GBIF].

Phoma trabutiana Sacc. Syll. fung. (Abellini) 3: 114 (1884)

Ref.: Saccardo (1884), Roumeguère (1887b), Kedad \& Bouznad (2018).

Phoma tricolor (Durieu \& Mont.) Sacc. Syll. fung. (Abellini) 3: 144 (1884)

Ref.: Saccardo (1884).

Phoma vicina f. platani Sacc. Revue mycol., Toulouse 3(no. 9): 28 (1881)

Ref.: Saccardo \& Roumeguère (1881), Saccardo (1884), Kedad \& Bouznad (2018).

Phoma yuccicola Pat. C. r. Congr. Soc. sav. Paris: 254 (1909) [1908]

Ref.: Saccardo (1913), Pfister (1977).

Stagonosporopsis cucurbitacearum (Fr.) Aveskamp, Gruyter \& Verkley. in Aveskamp, Gruyter, Woudenberg, Verkley \& Crous, Stud. Mycol. 65: 45 (2010)

syn. Didymella bryoniae (Fuckel) Rehm, Ber. naturhist. Augsburg 26: 27 (1881)

Ref.: Kedad \& Bouznad (2018).

\section{Didymosphaeriaceae}

Didymosphaeria celata (Curr.) Sacc. Syll. fung. (Abellini) 1: 705 (1882)

Ref.: Roumeguère (1885), Saccardo \& Roumeguère (1885), Aptroot (1998), Kedad \& Bouznad (2018), [BR], [GBIF].

Didymosphaeria costata Duvernoy \& Maire. Bull. Soc. Hist. nat. Afr. N. 13(1): 27 (1922)

Ref.: Kedad \& Bouznad (2018), [MPU], [GBIF].

Didymosphaeria diplodioides Sacc. Syll. fung. (Abellini) 1: 703 (1882)

syn. Sphaeria diplodioides P. Crouan \& H. Crouan, Florule Finistère (Paris): 26 (1867)

Ref.: [USDA]
Didymosphaeria echinospora Faurel \& Schotter. Mémoire, Institut de Recherches Sahariennes, Université d'Alger 4: 54 (1958)

Ref.: Aptroot (1998).

Didymosphaeria epidermidis (Fr.) Fuckel. Jb. nassau. Ver. Naturk. 23-24: 140 (1870) [1869-70]

syn. Sphaeria epidermidis Fr., Syst. mycol. (Lundae) 2(2): 499 (1823)

Ref.: Saccardo \& Berlese (1886a), Kedad \& Bouznad (2018), [GBIF], Durieu (1846), [P].

Kalmusia variispora (Verkley, Göker \& Stielow) Ariyaw. \& K.D. in Ariyawansa, Tanaka, Thambugala, Phookamsak, Tian \& Campo, Fungal Diversity 68: 85 (2014)

syn. Dendrothyrium variisporum Verkley, Göker \& Stielow, in Verkley, Dukik, Renfurm, Göker \& Stielow, Persoonia 32: 36 (2014)

Ref.: Teponno et al. (2017).

Montagnula longipes (Trab.) Aptroot Nova Hedwigia 60(3-4): 339 (1995)

syn. Didymosphaerella longipes (Trab.) Cooke, Grevillea 18(no. 86): 29 (1889)

Ref.: Saccardo (1886a \& 1891), Roumeguère (1887b), Maire (1916d), Maire \& Werner (1937), Aptroot (1995), Kedad \& Bouznad (2018), [GBIF], [USDA].

Montagnula opulenta (de Not.) Aptroot. Nova Hedwigia 60(3-4): 340 (1995)

syn. Sphaeria opulenta De Not., Mém. R. Accad. Sci. Torino, Ser. 2 3: 64 (1841)

Ref.: Durieu (1846), Kedad \& Bouznad (2018), [P].

Montagnula phragmospora (Durieu \& Mont.) Crivelli. Ueber die Heterogene Ascomycetengattung Pleospora Rabh.; Vorschlag für eine Aufteilung (Diss. Eid genössischen Technischen Hochschule Zürich 7318): 155 (1983)

syn. Pleospora phragmospora (Durieu \& Mont.) Ces. $\&$ De Not., in Rabenhorst, Fungi europ. exsicc.: no. 1543 (1873)

Ref.: Saccardo (1883), Berlese (1892-1905), [GBIF].

Montagnula gigantea (Mont.) Berl. Icon. fung. (Abellini) 2(2-3): 69 (1896)

Ref.: Berlese (1892-1905).

Pseudopithomyces chartarum (Berk. \& M.A. Curtis) Jin F. Li, Ariyaw. in Ariyawansa et al., Fungal Diversity: 10.1007/s13225-015-0346-5, [40] (2015)

syn. Pithomyces chartarum (Berk. \& M.A. Curtis) M.B. Ellis, Mycol. Pap. 76: 13 (1960) 
Ref.: Locquin-Linard (1988).

Pseudopithomyces sacchari (Speg.) Ariyaw. \& K.D. Hyde. in Ariyawansa et al., Fungal Diversity: 10.1007/s 13225-015-0346-5, [43] (2015)

syn. Pithomyces sacchari (Speg.) M.B. Ellis, Mycol. Pap. 76: 17 (1960)

Ref.: [P].

\section{Dothideaceae}

Dothidea lentisci Durieu \& Mont. in Durieu, Expl. Sci. Alg., Fl. Algér. 1(livr. 14): 548 (1848) [1846-49]

Ref.: Durieu (1846), Kedad \& Bouznad (2018), [P].

Dothidea ventricosa Durieu \& Mont. Grevillea 20(no. 96): 107 (1885)

Ref.: [GBIF], [P].

Omphalospora melaena (Fr.) Höhn. Sber. Akad. Wiss. Wien, Math.-naturw. Kl., Abt. 1 128(7-8): 603 (1919)

syn. Dothidella spinicola Höhn., Annln K. K. naturh. Hofmus. Wien 20: 3 (1905)

Phoma melaena (Fr.) Mont. \& Durieu, Expl. Sci. Alg., Fl. Algér. 1: 437 (1849)

Ref.: Saccardo (1884), Andreansky (1934), Maire \& Werner (1937), Kedad \& Bouznad (2018).

Scirrhia rimosa (Alb. \& Schwein.) Fuckel. Jb. nassau. Ver. Naturk. 23-24: 221 (1870) [1869-70]

Ref.: Maire (1906), Maire (1915c), Kedad \& Bouznad (2018), [USDA], [MA], [GBIF], [USDA].

\section{Dothioraceae}

Dothiora oleae (DC.) Crous. in Crous \& Groenewald, Fungal Biology 120(11): 1409 (2016)

syn. Coleophoma oleae (DC.) Petr. \& Syd., Beih. Reprium nov. Spec. Regni veg. 42(1): 469 (1927) [1926]

Dothiora sphaeroides (Pers.) Fr. in Crous \& Groenewald, Fungal Biology 120(11): 1409 (2016)

syn. Dothidea sphaeroides (Pers.) Fr. [as 'sphaerioides'], Observ. mycol. (Havniae) 2: 348 (1818)

Ref.: Durieu (1846), Kedad \& Bouznad (2018), [GBIF], [P].

Sydowia polyspora (Bref. \& Tavel) E. Müll. Sydowia 7(5-6): 342 (1953)

Ref.: [NCBI].

\section{Elsinoaceae}

Elsinoe ampelina Shear. Phytopathology 19(7): 677 (1929)
Ref.: Kedad \& Bouznad (2018).

Sphaceloma erigens (Maire) Arx Verh. K. ned. Akad. Wet., tweede sect. 51(3): 85 (1957)

syn. Gloeosporium erigens Maire, Bull. Soc. Hist. nat. Afr. N. 7(8): 301 (1916)

Ref.: Maire (1916d), Saccardo (1931), Kedad \& Bouznad (2018), [MA], [MPU], [GBIF], [USDA]

\section{Eremomycetaceae}

Eremomyces bilateralis Malloch \& Cain Can. J. Bot. 49(6): 849 (1971)

Ref.: Locquin-Linard (1988).

\section{Gloniaceae}

Cenococcum geophilum Fr. Syst. mycol. (Lundae) 3(1): 66 (1829)

Ref.: Beddiar et al. (2015).

Cenococcum graniforme (Sowerby) Ferd. \& Winge. Kongelige Veter. Landbohogskol. Aarsskr.: 332-385 (1925)

Ref.: Nezzar-Hocine (1996 \& 1998), Kedad \& Bouznad (2018).

Glonium varium (Fr.) Sacc. Syll. fung. (Abellini) 2: 735 (1883)

syn. Hysterium varium Fr., Observ. mycol. (Havniae) 1: 192 (1815)

Ref.: Durieu (1846), Saccardo (1883), Kedad \& Bouznad (2018), [GBIF], [P].

\section{Halotthiaceae}

Halotthia posidoniae (Durieu \& Mont.) Kohlm. Nova Hedwigia 6: 9 (1963)

syn. Amphisphseria posidoniae, Sphaeria posidoniae

Ref: Durieu (1846), Montagne (1856), Saccardo (1882b), Maire (1915c), Kohlmeyer (1963), Kedad \& Bouznad (2018), [MA], [BR], [P], [S], [SVH], [GBIF].

\section{Hysteriaceae}

Gloniopsis orygmaea (Mont. \& Duby) Sacc. Syll. fung. (Abellini) 2: 773 (1883)

Ref.: Saccardo (1883), Kedad \& Bouznad (2018), Duby (1862), [GBIF], [P].

Gloniopsis praelonga (Schwein.) Underw. \& Earle. Bull. Alab. Agric. Exper. Stat. 80: 196 (1897)

syn. Gloniopsis decipiens De Not., G. bot. ital. 2(2): 23 (1847), Hysterium muelleri Duby, Mém. Soc. Phys. Hist. nat. Genève 16(1): 44 (1861) [1862]

Ref.: [MPU], [GBIF], [P]. 
Hysterium angustatum Alb. \& Schwein. Consp. fung. (Leipzig): 55 (1805)

Ref.: Maire Maire (1916), Kedad \& Bouznad (2018), [MA], [USDA]

Hysterium confluens Schwein. Trans. Am. phil. Soc., New Series 4(2): 245 (1832) [1834]

Ref.: [P].

Hysterium orygmaeum Mont. \& Duby, in Duby, Mém. Soc. Phys. Hist. nat. Genève 16(1): 31 (1861) [1862]

Ref.: Duby (1862), , [GBIF], [P].

Hysterium pulicare Pers. Neues Mag. Bot. 1: 85 (1794)

Ref.: Boivin (1840), Durieu (1846), Duby (1862), Kedad \& Bouznad (2018), [GBIF], [P].

Hysterium stenogramma Durieu \& Mont. in Durieu, Expl. Sci. Alg., Fl. Algér. 1(livr. 14): 553 (1848) [1846-49]

Ref.: Durieu (1846), Montagne (1856), Duby (1862), Saccardo (1883), Kedad \& Bouznad (2018), [GBIF], $[\mathrm{P}]$.

Hysterium vulgare de Not. G. bot. ital. 2(2): 18 (1847)

Ref.: Saccardo \& Roumeguère (1881), Saccardo (1883), Kedad \& Bouznad (2018).

Hysterobrevium curvatum (Fr.) Math. \& Granmo. Sluttrapport for artsprojektet sekksporresopper i Finnmark (Troms $\varnothing$ ): 25 (2012)

syn. Gloniopsis curvata (Fr.) Sacc., Syll. fung. (Abellini) 2: 775 (1883)

Hysterium curvatum (Fr.) Duby, Mém. Soc. Phys. Hist. nat. Genève 16(1): 42 (1861) [1862]

Ref.: Durieu (1846), Saccardo (1883), [GBIF], [P].

Hysterobrevium smilacis (Schwein.) E. Boehm \& C.L. Schoch. in Boehm, Mugambi, Miller, Huhndorf, Marincowitz, Spatafora \& Schoch, Stud. Mycol. 64: 63 (2009)

Ref.: Kedad \& Bouznad (2018).

\section{Lentitheciaceae}

Setoseptoria arundinacea (Sowerby) Kaz. Tanaka \& K. Hiray. in Tanaka, Hirayama, Yonezawa, Sato, Toriyabe, Kudo, Hashimoto, Matsumura, Harada, Kurihara, Shirouzu \& Hosoya, Stud. Mycol. 82: 101 (2015)

syn. Lentithecium arundinaceum (Sowerby) K.D. Hyde, J. Fourn. \& Ying Zhang, Fungal Diversity 38: 236 (2009)
Sphaeria godini Desm., Annls Sci. Nat., Bot., sér. 3 5: 49 (1846)

Ref.: Durieu (1846), Kedad \& Bouznad (2018).

\section{Leptosphaeriaceae}

Leptosphaeria castagnei (Durieu \& Mont.) Sacc. Fungi venet. nov. vel. Crit., Sér. 2: 317 (1875)

syn. Sphaeria castagnei Durieu \& Mont., in Durieu, Expl. Sci. Alg., Fl. Algér. 1(livr. 14): 528 (1848) [1846-49]

Ref.: Durieu (1846), Saccardo (1883), Kedad \& Bouznad (2018), [GBIF], [P].

Leptosphaeria crozalsiana Maire. Annls mycol. 11(4): 352 (1913)

Ref.: Maire (1913), Saccardo (1928), [MPU], [GBIF].

Leptosphaeria culmicola (Fr.) P. Karst. Bot. Ztg. 24: 412 (1866)

syn. Sphaeria culmicola Fr., Syst. mycol. (Lundae) 2(2): 430 (1823)

Ref.: [GBIF], [P].

Leptosphaeria culmifraga (Fr.) Ces. \& de Not. Comm. Soc. crittog. Ital. 1(fasc. 4): 235 (1863)

syn. Sphaeria culmifraga Fr., Syst. mycol. (Lundae) 2(2): 510 (1823)

Ref.: Durieu (1846), Kedad \& Bouznad (2018), [GBIF], [P].

Leptosphaeria debeauxii Sacc. \& Roum. in Saccardo, Michelia 2(no. 7): 318 (1881)

Ref.: Debeaux et al. (1880), Saccardo (1882a), Kedad \& Bouznad (2018), [BR], [NY], [S], [SVH], [SVH], [GBIF], [USDA]

Leptosphaeria duplex (Sowerby) Sacc. Syll. fung. (Abellini) 2: 87 (1883)

syn. Sphaeria duplex Sowerby, Col. fig. Engl. Fung. Mushr. (London) 3(no. 26): tab. 375, fig. 4 (1803)

Ref.: Boivin (1840), Kedad \& Bouznad (2018).

Leptosphaeria icositana Maire. Bull. Soc. Hist. nat. Afr. N. 8(7): 170 (1917)

Ref.: Maire (1917a), Saccardo (1928), Kedad \& Bouznad (2018), [MPU], [GBIF].

Leptosphaeria indeprensa (Durieu \& Mont.) Sacc. Syll. fung. (Abellini) 2: 85 (1883)

syn. Sphaeria indeprensa Durieu \& Mont., in Durieu, Expl. Sci. Alg., Fl. Algér. 1(livr. 14): 538 (1848) [1846-49] 
Ref.: Durieu (1846), Montagne (1856), Saccardo (1883), Kedad \& Bouznad (2018), [P], [GBIF], Berlese (1892-1905).

Leptosphaeria macrochloae Lacoste. Revue Mycol., Paris 22(suppl. colon.): 12 (1957)

Ref.: Lacoste (1957), CMI (1963).

Leptosphaeria obesa (Durieu \& Mont.) Sacc. Fungi italica autogr. del. 5-8: tab. 284 (1878)

syn. Heptameria obesa (Durieu \& Mont.) Sacc., Syll. fung. (Abellini) 2: 88 (1883), Sphaeria obesa Durieu \& Mont., in Durieu, Expl. Sci. Alg., Fl. Algér. 1(livr. 14): 526 (1849) [1846-49]

Ref.: Durieu (1846), Montagne (1856), Saccardo (1883), Berlese (1894), Kedad \& Bouznad (2018), Berlese (1892-1905).

Leptosphaeria phiala (Durieu \& Mont.) Sacc. Syll. fung. (Abellini) 2: 19 (1883)

syn. Sphaeria phiala Durieu \& Mont., in Durieu, Expl. Sci. Alg., Fl. Algér. 1(livr. 13): 519 (1848) [1846-49

Ref.: Durieu (1846), Saccardo (1883), Kedad \& Bouznad (2018), [P], [GBIF], Berlese (1892-1905).

Leptosphaeria stereicola Ellis. Am. Nat. 17: 317 (1883)

Ref.: [MPU], [GBIF].

Leptosphaeria stipae Trab. Étude sur 1'Halfa (Alger): 48 (1889)

Ref.: Saccardo (1928).

\section{Lophiostomataceae}

Cilioplea coronata (Niessl) Munk ex Crivelli. Ueber die Heterogene Ascomycetengattung Pleospora Rabh.; Vorschlag für eine Aufteilung (Diss. Eid genössischen Technischen Hochschule Zürich 7318): 172 (1983)

syn. Pleospora coronata Niessl, Verh. nat. Ver. Brünn 14: 176 (1876)

Pyrenophora coronata f. umbelliferarum Sacc., Revue mycol., Toulouse 3(no. 11): 9 (1881)

Ref.: Saccardo \& Roumeguère (1881), Saccardo (1883), Kedad \& Bouznad (2018), Berlese (18921905).

Lophidium fraudulentum (Durieu \& Mont.) Sacc. Syll. fung. (Abellini) 2: 715 (1883)

syn. Sphaeria fraudulenta Durieu \& Mont., in Durieu, Expl. Sci. Alg., Fl. Algér. 1(livr. 13): 505 (1848) [1846-49]

Ref.: Durieu (1846), Montagne (1856), Saccardo (1883), Kedad \& Bouznad (2018), [P], [GBIF], Berlese (1892-1905).
Lophidium pseudocompressum Sacc. \& Berl. in Saccardo, Syll. fung., Addit. I-IV (Abellini): 260 (1886)

Ref.: Saccardo (1886a), Kedad \& Bouznad (2018).

Lophidium psilogrammum (Durieu \& Mont.) Sacc. Michelia 1(no. 3): 340 (1878)

syn. Sphaeria psilogramma Durieu \& Mont., in Durieu, Expl. Sci. Alg., Fl. Algér. 1(livr. 13): 506 (1848) [1846-49]

Ref.: Durieu (1846), Montagne (1856), Saccardo (1883), Kedad \& Bouznad (2018), [GBIF], [P].

Lophidium subcompressum Sacc. \& Berl. Revue mycol., Toulouse 8(no. 29): 34 (1886)

Ref.: Saccardo \& Berlese (1886a).

Lophiostoma compressum (Pers.) Ces. \& de Not. Comm. Soc. crittog. Ital. 1(fasc. 4): 219 (1863)

syn. Sphaeria compressa Pers., Syn. meth. fung. (Göttingen) 1: 56 (1801)

Ref.: Durieu (1846), Kedad \& Bouznad (2018), [GBIF], [P].

Lophiostoma crista-galli (Durieu \& Mont.) Sacc. Michelia 1(no. 3): 339 (1878)

syn. Sphaeria crista-galli Durieu \& Mont., in Durieu, Expl. Sci. Alg., Fl. Algér. 1(livr. 13): 507 (1848) [1846-49]

Ref.: Durieu (1846), Montagne (1856), Saccardo (1883), Kedad \& Bouznad (2018), [P], [GBIF], Berlese (1892-1905).

Lophiostoma excipuliforme (Fr.) Ces. \& de Not. Comm. Soc. crittog. Ital. 1(fasc. 4): 219 (1863)

syn. Sphaeria excipuliformis Fr., Observ. mycol. (Havniae) 1: 177 (1815)

Ref.: Durieu (1846), Kedad \& Bouznad (2018), [P].

Lophiostoma macrostomum (Tode) Ces. \& de Not. Comm. Soc. crittog. Ital. 1(fasc. 4): 219 (1863)

syn. Sphaeria macrostoma Tode, Fung. mecklenb. sel. (Lüneburg) 2: 12 (1791)

Ref.: Durieu (1846), Kedad \& Bouznad (2018), [GBIF], [P].

Lophiostoma semiliberum (Desm.) Ces. \& de Not. Comm. Soc. crittog. Ital. 1(fasc. 4): 220 (1863)

syn. Sphaeria semilibera Desm., Annls Sci. Nat., Bot., sér. 3 6: 78 (1846)

Ref.: Durieu (1846), Kedad \& Bouznad (2018).

Platystomum aromaticum (Fabre) Sacc. \& D. Sacc. Syll. fung. (Abellini) 17: 889 (1905) 
syn. Lophidium aromaticum Fabre, Annls Sci. Nat., Bot., sér. 6 9: 100 (1879) [1878]

Ref.: Berlese (1894), Berlese (1892-1905).

Platystomum chamaeropis (Maire) Sacc. in Trotter, Syll. fung. (Abellini) 24(2): 1111 (1928)

syn. Lophidium chamaeropis Maire, Bull. Soc. Hist. nat. Afr. N. 5(9): 256 (1914)

Ref.: Maire (1914).

Sigarispora caulium (Fr.) Thambug., Wanas., Kaz. Tanaka \& K. D. Hyde. Index Fungorum 267: 1 (2015)

syn. Lophiostoma caulium (Fr.) Ces. \& De Not., Comm. Soc. crittog. Ital. 1(fasc. 4): 219 (1863), Sphaeria caulium Fr., Syst. mycol. (Lundae) 2(2): 509 (1823)

Ref.: Kedad \& Bouznad (2018), [GBIF], Durieu (1846), [P].

\section{Lophiotremataceae}

Lophiotrema stenogramma (Durieu \& Mont.) Sacc. Syll. fung. (Abellini) 2: 681 (1883)

syn. Sphaeria stenogramma Durieu \& Mont., in Durieu, Expl. Sci. Alg., Fl. Algér. 1(livr. 14): 541 (1848) [1846-49]

Ref.: Durieu (1846), Montagne (1856), Saccardo (1883), Kedad \& Bouznad (2018), Berlese (18921905).

\section{Macrodiplodiopsidaceae}

Macrodiplodiopsis desmazieri (Mont.) Petr. Annls mycol. 20(5/6): 343 (1922)

Ref.: Saccardo \& Roumeguère (1881), Kedad \& Bouznad (2018).

\section{Massariaceae}

Navicella pileata (Tode) Fabre. Annls Sci. Nat., Bot., sér. 6 9: 96 (1879) [1878]

Ref.: [GBIF].

\section{Massarinaceae}

Helminthosporium anomalum J.C. Gilman \& E.V. Abbott. Iowa St. Coll. J. Sci. 1: 321 (1927)

Ref.: Dubost (1966).

Helminthosporium densum Sacc. \& Roum. Revue mycol., Toulouse 3(no. 9): 29 (1881)

Ref.: Saccardo \& Roumeguère (1881), Saccardo (1882a), Kedad \& Bouznad (2018).

Helminthosporium genistae Fr. Syst. mycol. (Lundae) 3(2): 360 (1832)

Ref.: Durieu (1846), Kedad \& Bouznad (2018).
Helminthosporium solani Durieu \& Mont. in Durieu, Expl. Sci. Alg., Fl. Algér. 1(livr. 9): 356 (1849) [184649]

Ref.: Durieu (1846), Montagne (1856), Kedad \& Bouznad (2018), [GBIF], [P].

Helminthosporium stipae Trab. Étude sur l'Halfa (Alger): 49 (1889)

Ref.: Saccardo (1931).

Helminthosporium urophorum Durieu \& Mont. Annls Sci. Nat., Bot., sér. 3 4: 366 (1845)

Ref.: Durieu (1846), Montagne (1856), Kedad \& Bouznad (2018), [GBIF], [P].

Helminthosporium velutinum Link [as 'Helmisporium'], Mag. Gesell. naturf. Freunde, Berlin 3(1-2): 10 (1809)

Ref.: Durieu (1846), Kedad \& Bouznad (2018).

\section{Melanommataceae}

Aposphaeria lentisci (Mont. \& Durieu) Pat. Bull. soc. Hist. nat. Autun 17: 156 (1904)

syn. Perisporium lentisci Mont. \& Durieu, in Durieu, Expl. Sci. Alg., Fl. Algér. 1(livr. 15): 563 (1849) [1846-49]

Ref.: Durieu (1846), Montagne (1856), Saccardo (1882b), Kedad \& Bouznad (2018), [GBIF], [P].

Herpotrichia pinetorum (Fuckel) G. Winter. Rabenh. Krypt.-Fl., Edn 2 (Leipzig) 1.2: 208 (1885)

syn. Herpotrichia juniperi (Sacc.) Petr., Annls mycol. 23(1/2): 43 (1925)

Ref.: [NCBI].

Melanomma minervae Fabre. Annls Sci. Nat., Bot., sér. 6 9: 91 (1879) [1878]

Ref.: Saccardo \& Berlese (1886a), Patouillard (1903), Kedad \& Bouznad (2018).

Melanomma verrucaria (Fr.) Sacc. Syll. fung. (Abellini) 2: 107 (1883)

syn. Sphaeria verrucaria Fr., Syst. mycol. (Lundae) 2(2): 496 (1823)

Ref.: Durieu (1846), Kedad \& Bouznad (2018), [GBIF], [P].

Praetumpfia obducens (Schumach.) Jaklitsch \& Voglmayr. Sydowia 69: 91 (2017)

syn. Cucurbitaria obducens (Schumach.) Petr., Annls mycol. 25(3/4): 226 (1927), Sphaeria obducens Schumach., Enum. pl. (Kjbenhavn) 2: 159 (1803)

Ref.: Durieu (1846), Kedad \& Bouznad (2018).

\section{Melanopsaceae}


Melanops delilei (Durieu \& Mont.) Weese. Ber. dt. bot. Ges. 37: 94 (1919)

syn. Botryosphaeria delilei (Durieu \& Mont.) Sacc., Syll. fung. (Abellini) 1: 460 (1882), Dothidea delilei Durieu \& Mont., in Durieu, Expl. Sci. Alg., Fl. Algér. 1(livr. 14): 546 (1848) [1846-49]

Ref.: Durieu (1846), Montagne (1856), Saccardo (1882b), Kedad \& Bouznad (2018).

Melanops diplodioidea (Durieu \& Mont.) Weese. Ber. dt. bot. Ges. 37: 95 (1919)

syn. Botryosphaeria diplodioides (Durieu \& Mont.) Sacc., Syll. fung. (Abellini) 1: 462 (1882), Sphaeria diplodioides Durieu \& Mont., in Durieu, Expl. Sci. Alg., Fl. Algér. 1(livr. 12): 480 (1848) [1846-49]

Ref.: Durieu (1846), Montagne (1856), Saccardo (1882b), Kedad \& Bouznad (2018), [GBIF], [P].

Melanops sycophila (Durieu \& Mont.) Weese. Ber. dt. bot. Ges. 37: 94 (1919)

syn. Botryosphaeria syconophila (De Not.) Ces. \& De Not., Comm. Soc. crittog. Ital. 1(fasc. 4): 212 (1863), Dothidea sycophila Durieu \& Mont., in Durieu, Expl. Sci. Alg., Fl. Algér. 1(livr. 14): 545 (1849) [1846-49], Sphaeria syconophila De Not., Mém. R. Accad. Sci. Torino, Ser. 2 13: 100 (1853)

Ref.: Durieu (1846), Montagne (1856), Saccardo (1882b), Kedad \& Bouznad (2018), [P], [P], [GBIF], [P].

\section{Microsphaeropsidaceae}

Microsphaeropsis olivacea (Bonord.) Höhn. [as 'olivaceus'], Hedwigia 59(5): 267 (1917)

syn. Coniothyrium olivaceum Bonord., in Fuckel, Jb. nassau. Ver. Naturk. 23-24: 377 (1870) [1869-70]

Ref.: Saccardo \& Roumeguère (1881), Kedad \& Bouznad (2018).

\section{Microthyriaceae}

Microthyrium boivinii Mont. Annls Sci. Nat., Bot., sér. 4 5: 342 (1856)

Ref.: Kedad \& Bouznad (2018).

Microthyrium mauritanicum Durieu \& Mont. Syll. gen. sp. crypt. (Paris): 261 (1856)

Ref.: Montagne (1856), Saccardo (1883), Saccardo (1891), Kedad \& Bouznad (2018), [GBIF], [P].

Microthyrium microscopicum Desm. Annls Sci. Nat., Bot., sér. 2 15: 138 (1841)

Ref.: [GBIF], [P].

\section{Muyocopronaceae}

Muyocopron smilacis (de Not.) Sacc. Syll. fung. (Abellini) 2: 660 (1883)

syn. Microthyrium smilacis De Not., Mém. R. Accad. Sci. Torino, Ser. 2 7: 22 (1845)

Ref.: Saccardo (1882a), Saccardo (1883), [MPU], [GBIF], [P].

\section{Mycosphaerellaceae}

Ascospora erythrostoma (Durieu \& Mont.) Sacc. Syll. fung. (Abellini) 3: 496 (1884)

syn. Septoria erythrostoma Durieu \& Mont., Expl. Sci. Alg., Fl. Algér. 1(livr. 15): 591 (1849) [1846-49]

Ref.: Roumeguère (1882), Durieu (1846), Saccardo (1884, 1886b, 1906)), Stevenson (1926), Braun (2015), Kedad \& Bouznad (2018), [MIN], [BR], [MIN], [S], [SVH], [GBIF].

Cercospora anagyridis Pat. Bull. Soc. mycol. Fr. 19(3): 261 (1903)

Ref.: Saccardo (1906), Patouillard (1903), Kedad \& Bouznad (2018).

Cercospora barrasii Gonz. Frag. Trab. Mus. Nac. Cienc. Nat., Ser. Bot. 9: 68 (1916)

Ref.: Maire (1917a), Kedad \& Bouznad (2018), [MPU], [GBIF].

Cercospora beticola Sacc. Nuovo G. bot. ital. 8(2): 189 (1876)

Ref.: Kedad \& Bouznad (2018).

Cercospora capparis Sacc. [as 'capparidis'] Nuovo G. bot. ital. 8(2): 189 (1876)

Ref.: [MIN], [BR], [CABI], [GBIF].

Cercospora echiorum Maire. Bull. Soc. Hist. nat. Afr. N. 8(7): 193 (1917)

Ref.: Maire (1917a), Kedad \& Bouznad (2018), [MPU], [GBIF].

Cercospora heliotropii-bocconii Scalia Atti Accad. Giorn. di Sci. Natur., Catania, IV 15: 15 (1902).

Ref.: Maire (1919), Kedad \& Bouznad (2018), [MA], [GBIF], [USDA]

Cercospora melongenae Welles, Phytopathology 12: 63 (1922)

Ref.: Kedad \& Bouznad (2018).

Cercospora mercurialis Pass. in Thümen, Mycoth. Univ., cent. 8: no. 783 (1877)

Ref.: Patouillard (1903), Maire (1919), Kedad \& Bouznad (2018), [MA], [GBIF].

Cercospora penzigii Sacc. in Saccardo, Syll. fung. (Abellini) 15: 84 (1901) 
syn. Cercospora fumosa Penz., in Saccardo, Michelia 2(no. 8): 476 (1882)

Ref.: Stevenson (1926), [USDA]

Cercospora punctiformis Sacc. \& Roum. Revue mycol., Toulouse 3(no. 9): 29 (1881)

Ref.: Saccardo (1882a, 1886b), Saccardo \& Roumeguère (1885), Stevenson (1926), Kedad \& Bouznad (2018).

Cercospora roesleri (Catt.) Sacc. Michelia 2(no. 6): 128 (1880)

Ref.: Kedad \& Bouznad (2018), [USDA]

Cercospora scandens Sacc. \& G. Winter. Hedwigia 22: 14 (1883)

Ref.: Maire (1915c), Kedad \& Bouznad (2018), [MA], [S], [SVH], [GBIF], [USDA]

Cercospora solani Thüm. Hedwigia 19: 135 (1880)

Ref.: Maire (1919), Kedad \& Bouznad (2018), [MA], [GBIF], [USDA]

Cercospora violae Sacc. Nuovo G. bot. ital. 8(2): 187 (1876)

Ref.: Kedad \& Bouznad (2018).

Cercosporina echiorum (Maire) Sacc. n Trotter, Syll. fung. (Abellini) 25: 897 (1931)

Ref.: Saccardo (1931).

Depazea vagans (Fr.) Fr. Deutschl. Krypt.-Fl. (Leipzig) 1: 139 (1844)

Ref.: [P], [GBIF].

Fulvia fulva (Cooke) Cif., Atti Ist. bot. Univ. Lab. crittog. Pavia, sér. 5 10(1): 246 (1954)

syn. Cladosporium fulvum Cooke, Grevillea 12(no. 61): 32 (1883), Passalora fulva (Cooke) U. Braun \& Crous, in Crous \& Braun, CBS Diversity Ser. (Utrecht) 1: 453 (2003)

Ref.: Lakhdari (2017), Kedad \& Bouznad (2018).

Mycosphaerella chamaeropis (Traverso) Tomilin. Opredelitel' Gribov roda Mycosphaerella Johans: 56 (1979)

Ref.: [USDA]

Mycosphaerella crepidophora (Mont.) Rehm. Annls mycol. 5(2): 210 (1907)

syn. Sphaerella crepidophora (Mont.) Sacc., Syll. fung. (Abellini) 1: 479 (1882)

Ref.: Maire (1906), Kedad \& Bouznad (2018).

Mycosphaerella cuprea (Sacc.) Siemaszko Trudy Tiflissk. Bot. Sada 3: 136 (1923) syn. Sphaerella cuprea Sacc., Annls mycol. 7(5): 432 (1909)

Ref.: Maire (1919), Kedad \& Bouznad (2018), [MA], [MPU], [GBIF], [USDA]

Mycosphaerella eryngii (Fr. ex Duby) Johanson ex Oudem. Rév. Champ. Pays-Bas (Amsterdam) 2: 213 (1897)

syn. Sphaerella eryngii (Fr. ex Duby) Cooke, Handb. Brit. Fungi 2: 917 (1871)

Ref.: Patouillard (1903), Kedad \& Bouznad (2018).

Mycosphaerella filicum (Desm.) Starbäck. Bih. K. svenska VetenskAkad. Handl., Afd. 3 15(no. 2): 9 (1889)

syn. Sphaeria filicum Desm., Annls Sci. Nat., Bot., sér. 2 13: 187 (1840)

Ref.: Durieu (1846), Kedad \& Bouznad (2018).

Mycosphaerella hepaticarum (Pat.) Petr. Annls mycol. 32(5/6): 378 (1934)

syn. Stigmatea hepaticarum Pat., Bull. Soc. mycol. Fr. 21: 121 (1905)

Ref.: Patouillard (1902), Saccardo (1913), Pfister (1977).

Mycosphaerella japonica (Pass.) Tomilin. Nov. sist. Niz. Rast. 6: 121 (1970) [1969]

syn. Sphaerella japonica Pass., Atti Reale Accad. Lincei, Rendic., Sér. 4 3(1): 6 (1887)

Ref.: Patouillard (1903), Kedad \& Bouznad (2018).

Mycosphaerella maculiformis (Pers.) J. Schröt. in Cohn, Krypt.-Fl. Schlesien (Breslau) 3.2(3): 333 (1894) [1908]

syn. Sphaerella maculiformis (Pers.) Auersw., in Gonnermann \& Rabenhorst, Mycol. eur. Abbild. Sämmtl. Pilze Eur. 5-6: 5 (1869)

Ref.: Durieu (1846), Kedad \& Bouznad (2018), [GBIF], [P].

Mycosphaerella mori (Fuckel) F.A. Wolf. Elisha Mitchell scient. Soc. 51: 165 (1935)

Ref.: Kedad \& Bouznad (2018).

Mycosphaerella punctiformis (Pers.) Starbäck, Bih. K. svenska VetenskAkad. Handl., Afd. 3 15(no. 2): 9 (1889)

syn. Phyllosticta maculiformis Sacc., Michelia 2(no. 8): 538 (1882), Ramularia endophylla Verkley \& U. Braun, in Verkley, Crous, Groenewald, Braun \& Aptroot, Mycol. Res. 108(11): 1276 (2004) 
Ref.: Saccardo \& Roumeguère (1881), Roumeguère (1885, 1889), [BR], [GBIF].

Mycosphaerella pyrina (Ellis \& Everh.) J.H. Mill. Mycologia 33(1): 80 (1941)

syn. Sphaerella pyrina Ellis \& Everh., N. Amer. Pyren. (Newfield): 275 (1892)

Ref.: Kedad \& Bouznad (2018).

Mycosphaerella salicicola (Fuckel) Johanson ex Oudem. Rév. Champ. Pays-Bas (Amsterdam) 2: 210 (1897)

syn. Septoria salicicola (Fr.) Sacc., Michelia 1(no. 2): 171 (1878)

Ref.: Durieu (1846), [P].

Mycosphaerella stipicola Tomilin. Nov. sist. Niz. Rast. 3: 173 (1966)

syn. Sphaerella chlorina Sacc. \& Trab., Étude sur l'Halfa (Alger): 48 (1889)

Ref.: Saccardo (1928).

Neocercosporidium smilacis (Thüm.) U. Braun, C. Nakash., Videira \& Crous. in Videira, Groenewald, Nakashima, Braun, Barreto, de Wit \& Crous, Stud. Mycol. 87: 326 (2017)

syn. Cercospora smilacis Thüm., Inst. Rev. Cient. Litt., Coimbra 27: 14 (1879), Passalora smilacis (Thüm.) U. Braun, in Triebel, Arnoldia 14: 30 (1997)

Ref.: Braun (2015), [GBIF].

Neophloeospora maculans (Berenger) Videira \& Crous. in Videira, Groenewald, Nakashima, Braun, Barreto, de Wit \& Crous, Stud. Mycol. 87: 338 (2017)

syn. Phloeospora maculans (Bérenger) Allesch., Rabenh. Krypt.-Fl., Edn 2 (Leipzig) 1(6): 935 (1900) [1899]

Ref.: [USDA]

Neopseudocercosporella capsellae (Ellis \& Everh.) Videira \& Crous in Videira, Groenewald, Braun, Shin \& Crous, Stud. Mycol. 83: 86 (2016)

syn. Pseudocercospora capsellae (Ellis \& Everh.) M.J. Morris \& Crous, S. Afr. J. Bot. 60(6): 325 (1994)

Ref.: [CABI].

Ovularia doronici Sacc. Michelia 2(no. 8): 638 (1882)

syn. Ramularia doronici (Sacc.) Lindau, Rabenh. Krypt.-Fl., Edn 2 (Leipzig) 1.8(6): 517 (1906)

Ref.: Maire (1916), [MA], [CABI], [GBIF], [USDA]

Passalora ariae (Fuckel) U. Braun \& Crous. in Crous \& Braun, CBS Diversity Ser. (Utrecht) 1: 65 (2003)

Ref.: Roumeguère (1882).
Passalora deightonii (N. Pons \& B. Sutton) U. Braun $\&$ Crous. in Braun, Schlechtendalia 8: 36 (2002)

syn. Cercosporidium deightonii N. Pons \& B. Sutton, Mycol. Res. 100(7): 818 (1996)

Ref.: Pons \& Sutton (1996).

Pharcidia calcariae f. macrospora Vouaux. Bull. Soc. mycol. Fr. 28: 253 (1912)

Ref.: Saccardo (1928).

Pharcidia calcariae f. minor Vouaux. Bull. Soc. mycol. Fr. 28: 253 (1912)

Ref.: Saccardo (1928).

Phloeospora achyranthea Sacc. \& Berl. in Saccardo, Syll. fung. (Abellini) 10: 399 (1892)

Ref.: Saccardo \& Berlese (1886a), Saccardo (1886a), Saccardo (1892), Kedad \& Bouznad (2018).

Phloeospora bupleuri (Desm.) Höhn. Öst. bot. Z. 66: 107 (1916)

syn. Septoria bupleuri Desm., Annls Sci. Nat., Bot., sér. 3 11(2): 346 (1849)

Ref.: Durieu (1846), Montagne (1856), Kedad \& Bouznad (2018), [GBIF], [P].

Polythrincium trifolii Kunze. Mykologische Hefte (Leipzig) 1: 14 (1817)

syn. Phyllachora trifolii (Pers.) Fuckel, Jb. nassau. Ver. Naturk. 23-24: 218 (1870) [1869-70]

Sphaeria trifolii Pers., Syn. meth. fung. (Göttingen) 1: 30 (1801))

Ref.: Durieu (1846), Durieu (1846), Kedad \& Bouznad (2018), [MA], [P], [GBIF], Patouillard (1897b), Maire (1915c), [MA], [MA], [USDA]

Pseudocercospora bonjeaniae (Maire) U. Braun \& Crous. in Crous \& Braun, CBS Diversity Ser. (Utrecht) 1: 85 (2003)

syn. Cercospora bonjeaniae Maire, Bull. Soc. Hist. nat. Afr. N. 8(7): 193 (1917)

Ref.: Maire (1917a), Kedad \& Bouznad (2018).

Pseudocercospora ceratoniae (Pat. \& Trab.) Deighton. Mycol. Pap. 140: 141 (1976)

syn. Ref.: Pat. \& Trab., Bull. Soc. mycol. Fr. 19(3): 260 (1903)

Ref.: Saccardo (1906), Patouillard (1903), Stevenson (1926), Pfister (1977), Kedad \& Bouznad (2018).

Pseudocercospora cladosporioides (Sacc.) U. Braun. Mycotaxon 48: 282 (1993)

syn. Cercospora cladosporioides Sacc., Michelia 2(no. 8): 556 (1882) 
Ref.: Patouillard (1903), Stevenson (1926), Kedad \& Bouznad (2018), [CABI].

Pseudocercospora fuligena (Roldan) Deighton. Mycol. Pap. 140: 144 (1976)

Ref.: Kedad \& Bouznad (2018).

Pseudocercospora neriella (Sacc.) Deighton, Mycol. Pap. 140: 149 (1976)

Ref.: Benia \& Pujade-Villar (2014), Kedad \& Bouznad (2018).

Pseudocercospora pycnidioides (Chupp) U. Braun \& Crous. in Crous \& Braun, CBS Diversity Ser. (Utrecht) 1: 345 (2003)

Ref.: Kedad \& Bouznad (2018).

Ramularia anchusae C. Massal. Malpighia 8: 213 (1894)

Ref.: Maire (1916), Kedad \& Bouznad (2018), [MA], [MPU], [CABI], [GBIF], [USDA]

Ramularia ballotae C. Massal. Botan. Zbl. 42(no. 26): 386 (1890)

Ref.: Maire (1919), Kedad \& Bouznad (2018), [MA], [GBIF], [USDA]

Ramularia centranthi Brunaud. Act. Soc. linn. Bordeaux 41: 430 (1887)

Ref.: Maire (1917b), Kedad \& Bouznad (2018), [MA], [GBIF], [USDA]

Ramularia cynarae Sacc. Michelia 1(no. 5): 536 (1879)

Ref.: Maire (d), Kedad \& Bouznad (2018), [MA], [GBIF], [USDA]

Ramularia grevilleana (Tul. \& C. Tul. ex Oudem.) Jørst. Meld. Stat. Plantepat. Inst. Oslo 50: 17 (1945)

syn. Mycosphaerella fragariae (Tul. \& C. Tul.) Lindau, in Engler \& Prantl, Nat. Pflanzenfam., Teil. I (Leipzig) 1(1): 424 (1897)

Ref.: Kedad \& Bouznad (2018).

Ramularia obliqua (Cooke) Oudem., Ned. kruidk. Archf, 1 sér. 1: 262 (1873)

syn. Ovularia obliqua (Cooke) Oudem., Hedwigia 22: 85 (1883)

Ref.: Patouillard (1903), Kedad \& Bouznad (2018).

Ramularia parietariae Pass. in Rabenhorst, Fungi europ. exsicc.: no. 2066 (1876)

Ref.: Patouillard (1897a), Kedad \& Bouznad (2018).

Ramularia purpurascens G. Winter. Bolm Soc. broteriana, Coimbra, sér. 1 2: 50 (1884) [1883]
Ref.: Kedad \& Bouznad (2018).

Ramularia winteri Thüm. Hedwigia 20: 57 (1881)

Ref.: Maire (1916), Kedad \& Bouznad (2018), [MA], [GBIF], [USDA]

Rhabdospora alexandrina Chrestian \& Maire. Rev. Phytopathol. appl. 1: 185 (1913)

Ref.: Maire (1915c), Maire (1917a), Saccardo (1931), Kedad \& Bouznad (2018), [MA], [S], [SVH], [GBIF], [USDA]

Rhabdospora arisari Durieu \& Mont. in Durieu, Expl. Sci. Alg., Fl. Algér. 1(livr. 15): 594 (1849) [1846-49]

syn. Rhabdospora arisari Durieu \& Mont., in Durieu, Expl. Sci. Alg., Fl. Algér. 1(livr. 15): 594 (1849)

Ref.: Montagne (1856), Durieu (1846), Roumeguère (1882), [BR], [GBIF].

Rhabdospora cinerescens Durieu \& Mont. Syll. gen. sp. crypt. (Paris): 278 (1856)

Ref.: Montagne (1856).

Rhabdospora oleandri (Durieu \& Mont.) Sacc. Syll. fung. (Abellini) 3: 583 (1884)

syn. Septoria oleandri Durieu \& Mont., in Durieu, Expl. Sci. Alg., Fl. Algér. 1(livr. 15): 593 (1849) [1846-49]

Ref.: Durieu (1846), Montagne (1856), Saccardo (1884), Kedad \& Bouznad (2018).

Rhabdospora peregrina Durieu \& Mont. in Durieu, Expl. Sci. Alg., Fl. Algér. 1(livr. 15): 592 (1849) [1846-49]

Ref.: Saccardo (1884).

Rhabdospora rhamni Durieu \& Mont. in Durieu, Expl. Sci. Alg., Fl. Algér. 1(livr. 15): 596 (1849) [1846-49]

Ref.: Durieu (1846), Montagne (1856).

Ruptoseptoria unedonis (Roberge ex Desm.) Quaedvl., Verkley \& Crous. Stud. Mycol. 75: 357 (2013)

syn. Septoria unedonis Roberge ex Desm., Annls Sci. Nat., Bot., sér. 3 8: 20 (1847)

Ref.: Patouillard (1897a), Schneider-Orelli (1913), Stevenson (1926), Kedad \& Bouznad (2018).

Septoria acanthi Thüm. J. Sci. math. phys. nat. Lisboa, 1 Ser. 6(nos 21-24): 252 (1878)

Ref.: Saccardo \& Berlese (1886a), Maire (1916), Stevenson (1926), Maire \& Werner (1937), Kedad \& Bouznad (2018), [MA], [MPU], [GBIF], [USDA] 
Septoria ampelina Berk. \& M.A. Curtis. N. Amer. Fung.: no. 440 (1874)

Ref.: Kedad \& Bouznad (2018).

Septoria apiicola Speg. Boln Acad. nac. Cienc. Córdoba 11(2): 294 (1887) [1888]

Ref.: Kedad \& Bouznad (2018), [S], [SVH].

Septoria arethusa Penz. Fung. Agrum.: 510 (1882)

Ref.: Stevenson (1926).

Septoria ari Desm., Funghi Parm. Septor.: no. 125 (1847)

Ref.: Patouillard (1897a), Stevenson (1926), Maire \& Werner (1937), Kedad \& Bouznad (2018).

Septoria asperulae-arvensis Maire. Bull. Soc. Hist. nat. Afr. N. 8(7): 181 (1917)

Ref.: Maire (1917a, 1917b), Maire, Kedad \& Bouznad (2018), [MA], [MPU], [GBIF], [USDA]

Septoria bellevaliae Pat. Bull. Soc. mycol. Fr. 21: 121 (1905)

Ref.: Patouillard (1902), Saccardo (1913), Stevenson (1926), Pfister (1977), Kedad \& Bouznad (2018).

Septoria cinerascens Durieu \& Mont. in Durieu, Expl. Sci. Alg., Fl. Algér. 1(livr. 15): 592 (1849) [1846-49]

Ref.: Durieu (1846), Montagne (1856).

Septoria cirrosae Sacc. Fungi Imperfecti exs., Fasc.: no. 761 (1913)

Ref.: Maire (1915c), Kedad \& Bouznad (2018), [MA], [MPU], [S], [SVH], [GBIF], [USDA]

Septoria citri Pass. in Thümen, Mycoth. Univ., cent. 1: no. 13 (1877)

Ref.: [USDA]

Septoria convolvuli Desm. Annls Sci. Nat., Bot., sér. 2 17: 108 (1842)

Ref.: Durieu (1846), Maire (1915c), Kedad \& Bouznad (2018), [MA], [GBIF], [USDA]

Septoria convolvuli Desm. Annls Sci. Nat., Bot., sér. 217: 108 (1842)

Ref.: Durieu (1846), Maire (1915c), Kedad \& Bouznad (2018), [MA], [GBIF], [USDA]

Septoria cyclaminis Durieu \& Mont. in Montagne, Syll. gen. sp. crypt. (Paris): 279 (1856)

Ref.: Durieu (1846), Montagne (1856), Stevenson (1926), Kedad \& Bouznad (2018).

Septoria dulcamarae Desm. Annls Sci. Nat., Bot., sér. 215: 135 (1841)

Ref.: Kedad \& Bouznad (2018).
Septoria eucalypti G. Winter \& Roum. Revue mycol., Toulouse 9(no. 33): 41 (1887)

Ref.: Roumeguère (1887a), Roumeguère (1887b), Stevenson (1926), Kirk (1985).

Septoria ficariae Desm. Annls Sci. Nat., Bot., sér. 2 15: 135 (1841)

Ref.: Durieu (1846), Kedad \& Bouznad (2018).

Septoria flammulae Pass. Funghi Parm. Septor.: no. 2 (1879)

Ref.: Kedad \& Bouznad (2018), [MPU], [GBIF].

Septoria gladioli Pass. in Rabenhorst, Fungi europ. exsicc.: no. 1956 (1875)

Ref.: Roumeguère (1886), [BR], [CABI], [GBIF].

Septoria hederae Desm. Annls Sci. Nat., Bot., sér. 2 19: 340 (1843)

Ref.: Durieu (1846), Kedad \& Bouznad (2018).

Septoria hyoseridis Maire. Bull. Soc. bot. Fr. 53: CLXXXVIII (1907) [1906]

Ref.: Maire (1906), Kedad \& Bouznad (2018).

Septoria limonum Pass. Atti Soc. Crittogam. Ital., Sér. 2: 23 (1879)

Ref.: Patouillard (1903), Kedad \& Bouznad (2018).

Septoria mortolensis Penz. \& Sacc. Atti Inst. Veneto Sci. lett., ed Arti, Sér. 6 2(5): 651 (1884)

Ref.: Saccardo (1884).

Septoria nitidula Durieu \& Mont. in Durieu, Expl. Sci. Alg., Fl. Algér. 1(livr. 15): 593 (1849) [1846-49]

Ref.: Durieu (1846), Saccardo (1884), Kedad \& Bouznad (2018).

Septoria oleae Durieu \& Mont. in Durieu, Expl. Sci. Alg., Fl. Algér. 1(livr. 15): 590 (1849) [1846-49]

Ref.: Durieu (1846), Kedad \& Bouznad (2018).

Septoria peregrina Sacc. G. bot. ital., n.s. 23(2): 218 (1916)

Ref.: Durieu (1846), Kedad \& Bouznad (2018).

Septoria petroselini Desm. Mém. Soc. des Sc. de Lille: 97 (1843)

Ref.: Maire (1915c, 1916), Kedad \& Bouznad (2018), [MA], [GBIF], [USDA].

Septoria petroselini var. apii Briosi \& Cavara, Funghi Parass. Piante Colt. od Utili: no. 144 (1891)

Ref.: [MA]

Septoria quercina Desm. Annls Sci. Nat., Bot., sér. 3 8: 25 (1847)

Ref.: Durieu (1846), Kedad \& Bouznad (2018). 
Septoria rhamni-catharticae Ces. in Rabenhorst, Klotzschii Herb. Viv. Mycol., Edn 2: no. 1956 (1880) Ref.: [CABI].

Septoria rhynchosporae Durieu \& Mont. in Durieu, Expl. Sci. Alg., Fl. Algér. 1(livr. 15): 594 (1849) [1846-49]

Ref.: Durieu (1846), Saccardo (1884), Kedad \& Bouznad (2018).

Septoria rosae var. sempervirentis Durieu \& Mont. Pl. Crypt. Nord France, Edn 1: no. 535 (1831)

Ref.: [MPU].

Septoria saccardoana Roum. Revue mycol., Toulouse 4(no. 13): 22 (1882)

Ref.: Saccardo (1884), Kedad \& Bouznad (2018), [BR], [NY], [GBIF].

Septoria scillae Westend. in Kickx, Not. 3: 18 (1874)

Ref.: Patouillard (1897a), Kedad \& Bouznad (2018).

Septoria smilacina Durieu \& Mont. in Durieu, Expl. Sci. Alg., Fl. Algér. 1(livr. 15): 591 (1849) [1846-49]

Ref.: Durieu (1846), Montagne (1856), Saccardo (1884), Patouillard (1897b), Kedad \& Bouznad (2018).

Septoria stellariae Roberge ex Desm. Annls Sci. Nat., Bot., sér. 3 8: 22 (1847)

Ref.: Maire (1916), Kedad \& Bouznad (2018), [MA], [GBIF], [USDA]

Septoria stipae Trab. Étude sur l'Halfa (Alger): 49 (1889)

Ref.: Saccardo (1931).

Septoria umbilici Maire. Bull. Soc. Hist. nat. Afr. N. 8(7): 182 (1917)

Ref.: Maire (1917a), Saccardo (1931), Kedad \& Bouznad (2018), [MPU], [GBIF].

Septoria unedonis var. vellanensis Briosi \& Cavara. Funghi Parass. Piante Colt. od Utili, Fasc. 5: no. 121 (1890)

Ref.: Schneider-Orelli (1913).

Septoria xanthii Desm. Annls Sci. Nat., Bot., sér. 3 8: 25 (1847)

Ref.: Maire (1917b), Kedad \& Bouznad (2018), [MA], [GBIF], [USDA]

Sphaerella gnidii Maire. Bull. Soc. Hist. nat. Afr. N. 8(7): 167 (1917)

Ref.: Maire (1917a), Saccardo (1928), Stevenson (1926), Kedad \& Bouznad (2018), [MPU], [GBIF].

Sphaerella hertiae Pat. Bull. Soc. mycol. Fr. 18(2): 52 (1902)
Ref.: Saccardo (1905), Pfister (1977), Kedad \& Bouznad (2018).

Sphaerella ornithogali (Grognot) Roum. Fung. Sel. Exs.: no. 4443 (1888)

Ref.: [BR], [GBIF], Roumeguère (1882).

Sphaerulina coronillae-junceae Bubák \& Gonz. Frag. in Bubák, Hedwigia 57: 5 (1915)-

Ref.: Maire \& Werner (1937).

Sphaerulina myriadea (DC.) Sacc. Michelia 1(no. 4): 399 (1878)

syn. Sphaeria myriadea DC., Fl. franç., Edn 3 (Paris)

5/6: 145 (1815)

Ref.: Durieu (1846), [GBIF], [P].

Sphaerulina myriadea var. viburni (Durieu \& Mont.) Sacc. Syll. fung. (Abellini) 2: 186 (1883)

Ref.: Saccardo (1883).

Sphaerulina rehmiana Jaap. Verh. bot. Ver. Prov. Brandenb. 52: 12 (1910)

syn. Septoria rosae Desm., Pl. Crypt. Nord France, Edn 1: no. 535 (1831)

Ref.: Durieu (1846), [GBIF].

Sphaerulina serograpta (Durieu \& Mont.) Sacc. Syll. fung. (Abellini) 2: 187 (1883)

syn. Sphaeria serograpta Durieu \& Mont., in Durieu, Expl. Sci. Alg., Fl. Algér. 1(livr. 14): 537 (1848) [1846-49]

Ref.: Durieu (1846), Montagne (1856), Saccardo (1883), Berlese (1894), Berlese (1892-1905), Kedad \& Bouznad (2018).

Stigmina carpophila (Lév.) M.B. Ellis. Mycol. Pap. 72: 56 (1959)

syn. Ascospora beijerinckii Vuill., J. Bot., Paris 2: 255 (1888), Clasterosporium carpophilum (Lév.) Aderh., Landwirtschaftliche Jahrbucher 30: 815 (1901)

Ref.: Maire (1906), Schneider-Orelli (1913), Kedad \& Bouznad (2018), [CABI].

Stigmina sapii (J. Miyake) M.B. Ellis. Mycol. Pap. 111: 42 (1967)

syn. Cercospora sebiferae Pat., Bull. Soc. mycol. Fr. 31: 78 (1915)

Ref.: Pfister (1977).

\section{Myriangiaceae}

Myriangium duriaei Mont. \& Berk. in Berkeley, London J. Bot. 4: 73 (1845)

Ref.: Maire (1915c), Kedad \& Bouznad (2018), [MA], [SVH], [GBIF], [USDA]

\section{Patellariaceae}


Patellaria atrata (Hedw.) Fr. Syst. mycol. (Lundae) 2(1): 158 (1822)

syn. Lecanidion atratum (Hedw.) Endl., Flora Pason 1: 46 (1830)

Ref.: Saccardo \& Roumeguère (1881), Patouillard (1897b), Kedad \& Bouznad (2018).

Rhytidhysteron rufulum (Spreng.) Speg. Anal. Soc. cient. argent. 90(3-6): 177 (1921) [1920]

Ref.: [NCBI].

\section{Phaeosphaeriaceae}

Ampelomyces quisqualis Ces. in Klotzsch, Bot. Ztg. 10: 301 (1852)

Ref.: Kedad \& Bouznad (2018).

Chaetoplea inverecunda (de Not.) Checa. Mycotaxon 68: 215 (1998)

syn. Sphaeria inverecunda De Not., Mém. R. Accad. Sci. Torino, Ser. 2 13: 102 (1853) [1846-49], Teichospora inverecunda (De Not.) Sacc., Syll. fung. (Abellini) 2: 293 (1883)

Ref.: Durieu (1846), Saccardo \& Roumeguère (1881), Saccardo (1883), Berlese (1892-1905), Kedad \& Bouznad (2018), [BR], [GBIF], [P].

Darluca ascochytoides Sacc. \& Roum. Mycotaxon 68: 215 (1998)

Ref.: Saccardo \& Roumeguère (1881), Saccardo (1884), Kedad \& Bouznad (2018).

Eudarluca caricis (Fr.) O.E. Erikss. Bot. Notiser 119: 33 (1966)

Ref.: [CABI].

Hendersonia macrochloae Trab. Étude sur l'Halfa (Alger): 49 (1889)

Ref.: Saccardo (1931), Lacoste (1957).

Hendersonia nobilis Durieu \& Mont. in Durieu, Expl. Sci. Alg., Fl. Algér. 1(livr. 15): 570 (1849) [1846-49]

Ref.: Durieu (1846), Montagne (1856), Roumeguère (1882), Saccardo (1884), Kedad \& Bouznad (2018), [BR], [P], [S], [GBIF].

Hendersonia peregrina Fautrey. Revue mycol., Toulouse 14(no. 53): 8 (1892)

Ref.: Roumeguère (1892), Saccardo (1892), Kedad \& Bouznad (2018).

Hendersonia sarmentorum Westend. Bull. Acad. R. Sci. Belg., Cl. Sci. 18(2): no. 60 (1852)

Ref.: [P].

Hendersonia stipae-pennatae Fautrey. Revue mycol., Toulouse 11(no. 43): 134 (1889)

Ref.: Lacoste (1957).
Neosetophoma italica W.J. Li, Camporesi \& K.D. Hyde in Liu et al., Fungal Diversity: 10.1007/s13225015-0324-y, [142] (2015)

Ref.: [NCBI].

Neosetophoma samarorum (Desm.) Gruyter, Aveskamp \& Verkley. in de Gruyter, Woudenberg, Aveskamp, Verkley, Groenewald \& Crous, Mycologia 102(5): 1075 (2010)

syn. Phoma samarorum Desm., Pl. Crypt. Nord France, Edn 1 7: no. 349 (1828)

Ref.: Patouillard (1903), Kedad \& Bouznad (2018).

Parastagonospora nodorum (Berk.) Quaedvl., Verkley \& Crous. Stud. Mycol. 75: 363 (2013)

syn. Stagonospora nodorum (Berk.) E. Castell. \& Germano, Annali Fac. Sci. agr. Univ. Torino 10: 71 (1977) [1975-76]

Ref.: Benbelkacem et al. (2011), Kedad \& Bouznad (2018).

Phaeosphaeria tini (Ellis \& Everh.) Huhndorf. in Barr, Huhndorf \& Rogerson, Mem. N. Y. bot. Gdn 79: 98 (1996)

syn. Leptosphaeria tini Ellis \& Everh., J. Mycol. 4(7): 64 (1888)

Ref.: Maire (1906), Kedad \& Bouznad (2018).

Phaeosphaeriopsis glaucopunctata (Grev.) M.P.S. Câmara, M.E. Palm \& A.W. Ramaley [as 'glaucopunctata'], in Câmara, Ramaley, Castlebury \& Palm, Mycol. Res. 107(5): 519 (2003)

syn. Leptosphaeria rusci (Fr.) Sacc., Syll. fung. (Abellini) 2: 74 (1883), Sphaeria rusci (Fr.) Wallr., Fl. crypt. Germ. (Norimbergae) 2: 776 (1833)

Ref.: Durieu (1846), Jaczewski (1893), Kedad \& Bouznad (2018), [MPU], [GBIF], [P].

Sclerostagonospora sessilis (Mont.) E. Gallego. in Gallego, Honrubia \& Díaz-Espejo, Int. J. Mycol. Lichenol. 3(1): 73 (1986)

syn. Hendersonia sessilis Mont., Annls Sci. Nat., Bot., sér. 3 11: 44 (1849)

Ref.: [MPU], [GBIF].

Sphaerellopsis filum (Biv.) B. Sutton. Mycol. Pap. 141: 196 (1977)

syn. Darluca filum (Biv.) Castagne, Outl. Brit. Fung. (London): 318 (1860)

Ref.: Saccardo \& Roumeguère (1881), Maire (1906), Battandier et al. (1914), Kedad \& Bouznad (2018).

Stagonospora agaves (Maubl.) Mouch. in Mouchacca \& Saint-Jore, Mycotaxon 74(2): 324 (2000)

syn. Hendersonia agaves Maubl., Bull. Soc. mycol. Fr. 19(3): 294 (1903) 
Ref.: Saccardo (1906), Maublanc (1903), Stevenson (1926), Mouchacca (2000), Kedad \& Bouznad (2018).

Stagonospora kentiae Maubl. Bull. Soc. mycol. Fr. 19(3): 293 (1903)

Ref.: Saccardo (1906), Maublanc (1903), Stevenson (1926), Mouchacca \& Saint-Jore (2000), Mouchacca (2000), K ekad \& Bouznad (2018).

Phaeotrichaceae

Trichodelitschia bisporula (P. Crouan \& H. Crouan) E. Müll. \& Arx. Beitr. Kryptfl. Schweiz 11(no. 2): 350 (1962)

syn. Pleurage minuta (Fuckel) Kuntze, Revis. gen. pl. (Leipzig) 3(3): 505 (1898)

Ref.: Faurel \& Schotter (1964), Locquin-Linard (1988).

\section{Phyllostictaceae}

Phyllosticta agaves Maubl. Bull. Soc. mycol. Fr. 19(3): 293 (1903)

Ref.: Saccardo (1906), Maublanc (1903), Stevenson (1926), Mouchacca (2000), Kedad \& Bouznad (2018).

Phyllosticta araliae Sacc. \& Berl. Revue mycol., Toulouse 8(no. 29): 35 (1886)

Ref.: Saccardo (1886a, 1882), Stevenson (1926), Kedad \& Bouznad (2018).

Phyllosticta asclepiadearum Westend. Bull. Acad. R. Sci. Belg., Cl. Sci. 18(2): 398 (1852)

Ref.: Roumeguère (1885), [GBIF].

Phyllosticta australis Speg. Anal. Soc. cient. argent. 10(1): 28 (1880)

Ref.: Kedad \& Bouznad (2018).

Phyllosticta caulicola Pat. Bull. Soc. mycol. Fr. 13: 213 (1897)

Ref.: Patouillard (1897a), Saccardo (1899), Stevenson (1926), Pfister (1977), Kedad \& Bouznad (2018).

Phyllosticta ceratoniae Berk. in Welwitsch, Some Notes upon the Cryptogamic Portion of the Plants collected in Portugal 1842-1850 by Dr Fried. Welwitsch, The Fungi: 8 (1853)

Ref.: [GBIF].

Phyllosticta citricarpa (McAlpine) Aa. Stud. Mycol. 5: 40 (1973)

syn. Phoma citricarpa McAlpine, Fungus Diseases of Citrus trees in Australia: 21 (1899)

Ref.: Maire (1934).

Phyllosticta debeauxii (Roum.) Moesz [as 'debauxii'], in Degen, Flora Velebitica 3: 293 (1938)

syn. Phoma debeauxii Roum., Revue mycol., Toulouse 4(no. 16): 216 (1882)

Ref.: Roumeguère (1882), Saccardo (1884), Patouillard (1897b), Kedad \& Bouznad (2018), [BR], [S], [SVH], [GBIF].

Phyllosticta eucalypti Thüm. Hedwigia 19: 180 (1879) Ref.: Magnani (1964), Stevenson (1926), Gibson (1975), Crous et al. (1989).

Phyllosticta ficariae Maire. Bull. Soc. Hist. nat. Afr. N. 8(7): 180 (1917)

Ref.: Maire (1917a), Saccardo (1931), Kedad \& Bouznad (2018), [MPU], [GBIF].

Phyllosticta fulvomaculans Maire. Bull. Soc. Hist. nat. Afr. N. 36(3): 41 (1945)

Ref.: Kedad \& Bouznad (2018).

Phyllosticta fuscozonata Thüm. Boll. Soc. Adriatica Sci. Nat. Triests 6: 135 (1880)

Ref.: Stevenson (1926), [GBIF], Roumeguère (1888a).

Phyllosticta hesperidearum (Catt.) Penz. Fung. Agrum.: 41 (1882)

Ref.: Patouillard (1903), Stevenson (1926), Kedad \& Bouznad (2018).

Phyllosticta microsticta Durieu \& Mont. Expl. Sci. Alg., Fl. Algér. 2(livr. 20): 612 (1855)

Ref.: Montagne (1856), Saccardo (1884), Kedad \& Bouznad (2018).

Phyllosticta nitidula Durieu \& Mont. in Montagne, Syll. gen. sp. crypt. (Paris): 279 (1856)

Ref.: Montagne (1856), Saccardo (1884), Stevenson (1926), Kedad \& Bouznad (2018).

Phyllosticta oleae Petri. Atti Reale Accad. Lincei, Rendic., Sér. 5 18(2): 636 (1909)

Ref.: Kedad \& Bouznad (2018).

Phyllosticta palmarum Rabenh. Fungi europ. exsicc., Edn nova, Ser. sec., Cent. 2: no. 2161 (1876)

Ref.: [GBIF].

Phyllosticta patouillardii Sacc. \& D. Sacc. Syll. fung. (Abellini) 18: 225 (1906)

Ref.: Saccardo (1906), Patouillard (1903), Pfister (1977), Kedad \& Bouznad (2018), [MPU], [GBIF].

Phyllosticta philoprina (Berk. \& M.A. Curtis) Wikee \& Crous. Stud. Mycol. 76: 19 (2013)

Ref.: [GBIF].

Phyllosticta pustulosa Sacc. \& Roum. Revue mycol., Toulouse 3(no. 9): 28 (1881)

Ref.: Saccardo \& Roumeguère (1881), Saccardo (1884), Kedad \& Bouznad (2018). 
Phyllosticta ruscicola Durieu \& Mont. Expl. Sci. Alg., Fl. Algér. 2(livr. 20): 611 (1849) [1846-49]

Ref.: Montagne (1856), Saccardo (1884), Maire \& Werner (1937).

\section{Piedraiaceae}

Memnonium schoenophilum Durieu \& Mont. in Durieu, Expl. Sci. Alg., Fl. Algér. 1(livr. 9): 351 (1848) [1846-49]

Ref.: Kedad \& Bouznad (2018).

\section{Planistromellaceae}

Kellermania macrospora (Durieu \& Mont.) Minnis \& A.H. Kenn. in Minnis, Kennedy, Grenier, Palm \& Rossman, Persoonia 29: 19 (2012)

syn. Septoria macrospora Durieu \& Mont., in Montagne, Syll. gen. sp. crypt. (Paris): 277 (1856), Stagonospora macrospora (Durieu \& Mont.) Sacc., Syll. fung. (Abellini) 3: 450 (1884)

Ref.: Durieu (1846), Montagne (1856), Saccardo (1884), Maire (1916), Maire \& Werner (1937), Kedad \& Bouznad (2018), [MA], [GBIF], [USDA]

\section{Pleomassariaceae}

Scolicosporium pauciseptatum Constant., Mycotaxon 41(2): 467 (1991)

syn. Hendersonia fusarioides Sacc., Michelia 1(no. 2): 213 (1878)

Ref.: Saccardo \& Roumeguère (1881), Saccardo (1884), Kedad \& Bouznad (2018).

\section{Pleosporaceae}

Alternaria allii Nolla. Phytopathology 17(2): 118 (1927)

Ref.: Kedad \& Bouznad (2018).

Alternaria alternata (Fr.) Keissl. Beih. bot. Zbl., Abt. 2 29: 434 (1912)

syn. Alternaria tenuis Nees, Syst. Pilze (Würzburg): 72 (1816) [1816-17]

Dubost (1966), Kachour (2004), Bouharati et al. (2008), Harzallah et al. (2009), Benzeghmane (2011), Belhoucine et al. (2011), Ladjal (2012), Bessadat et al. (2014), Kahoul et al. (2014), Bensaci et al. (2015), Bessadat et al. (2017), Rebbouh (2016), Kedad \& Bouznad (2018), Chamekh et al. (2019), [NCBI].

Alternaria arborescens E.G. Simmons. Mycotaxon 70: 356 (1999)

Ref.: Bessadat et al. (2014), Kedad \& Bouznad (2018), [NCBI].
Alternaria arbusti E.G. Simmons. Mycotaxon 48: 103 (1993)

Ref.: Simoneau \& Bessadat ([NCBI] 2019).

Alternaria atra (Preuss) Woudenb. \& Crous. Stud. Mycol. 75(1): 204 (2013)

syn. Ulocladium atrum Preuss, Linnaea 25: 75 (1852)

Ref.: Locquin-Linard (1988), Bekhouche et al. (1994), [P].

Alternaria botrytis (Preuss) Woudenb. \& Crous. Stud. Mycol. 75(1): 206 (2013)

syn. Ulocladium botrytis Preuss, Linnaea 24: 111 (1851)

Ref.: Locquin-Linard (1988), Belhoucine et al. (2011), Kedad \& Bouznad (2018).

Alternaria brassicae (Berk.) Sacc. Michelia 2(no. 6): 129 (1880)

Ref.: Kedad \& Bouznad (2018).

Alternaria cantlous (Yong Wang bis \& X.G. Zhang) Woudenb. \& C. Stud. Mycol. 75(1): 204 (2013)

Ref.: Simoneau \& Bessadat (2019), ([NCBI].

Alternaria chartarum Preuss Linnaea 24: 107 (1851) syn. Ulocladium chartarum (Preuss) E.G. Simmons, Mycologia 59(1): 88 (1967)

Ref.: Locquin-Linard (1988), Bekhouche et al. (1994), Kahoul et al. (2014), Chamekh et al. (2019), Kedad \& Bouznad (2018).

Alternaria consortialis (Thüm.) J.W. Groves \& S. Hughes. in Hughes, Can. J. Bot. 31: 636 (1953)

syn. Ulocladium consortiale (Thüm.) E.G. Simmons, Mycologia 59(1): 84 (1967)

Ref.: Nicot (1955), Locquin-Linard (1988), Bessadat et al. (2017), Kedad \& Bouznad (2018), Simoneau \& Bessadat (2019), [NCBI].

Alternaria crassa (Sacc.) Rands. Phytopathology 7: 337 (1917)

Ref.: Kedad \& Bouznad (2018), [NCBI].

Alternaria cucumerina (Ellis \& Everh.) J.A. Elliott. Am. J. Bot. 4: 472 (1917)

Ref.: Kedad \& Bouznad (2018).

Alternaria cumini E.G. Simmons. CBS Diversity Ser. (Utrecht) 6: 664 (2007)

Ref.: Simoneau \& Bessadat ([NCBI] 2019).

Alternaria dauci (J.G. Kühn) J.W. Groves \& Skolko. Canadian Journal of Research, Section C 22: 222 (1944)

Ref.: Bennichou (2010-11), Kedad \& Bouznad (2018), [NCBI]. 
Alternaria embellisia Woudenb. \& Crous. Stud. Mycol. 75(1): 191 (2013)

Ref.: Kedad \& Bouznad (2018).

Alternaria ethzedia E.G. Simmons. Mycotaxon 25(1): 300 (1986)

Ref.: [NCBI].

Alternaria graminicola E.G. Simmons. CBS Diversity Ser. (Utrecht) 6: 626 (2007)

Ref.: [NCBI].

Alternaria grandis E.G. Simmons. Mycotaxon 75: 96 (2000)

Ref.: Bessadat et al. (2016), Bessadat et al. (2017), Ayad et al. (2017), Kedad \& Bouznad (2018), [NCBI].

Alternaria humuli E.G. Simmons. Mycotaxon 83: 139 (2002)

Ref.: [NCBI].

Alternaria incomplexa E.G. Simmons. Mycotaxon 57: 394 (1996)

Ref.: [NCBI].

Alternaria infectoria E.G. Simmons. Mycotaxon 25(1): 298 (1986)

syn. Pleospora infectoria var. stipae Trab., Étude sur l'Halfa (Alger): 48 (1889))

Ref.: Saccardo (1928), Belhoucine et al. (2011), Kedad \& Bouznad (2018).

Alternaria lanuginosa (Harz) Sacc., Syll. fung. (Abellini) 4: 546 (1886

syn. Stemphylium lanuginosum Harz, Bull. Soc. Imp. nat. Moscou 44(1): 132 (1871), Ulocladium lanuginosum (Harz) E.G. Simmons, Mycologia 59(1): 80 (1967)

Ref.: Nicot (1955).

Alternaria linariae (Neerg.) E.G. Simmons. CBS Diversity Ser. (Utrecht) 6: 677 (2007)

Ref.: Bessadat et al. (2017), Ayad et al. (2017), Kedad \& Bouznad (2018), [NCBI].

Alternaria lolii (E.G. Simmons \& C.F. Hill) Woudenb. \& Crous. Stud. Mycol. 75(1): 193 (2013)

Ref.: [NCBI].

Alternaria metachromatica E.G. Simmons. Mycotaxon 50: 418 (1994)

Ref.: [NCBI].

Alternaria protenta E.G. Simmons. Mycotaxon 25(1): 207 (1986)

Ref.: Ayad et al. (2017), Kedad \& Bouznad (2018), [NCBI].
Alternaria radicina Meier, Drechsler \& E.D. Eddy. Phytopathology 12: 157 (1922)

Ref.: Kedad \& Bouznad (2018).

Alternaria smyrnii (P. Crouan \& H. Crouan) E.G. Simmons. Mycotaxon 55: 41 (1995)

Ref.: Kedad \& Bouznad (2018).

Alternaria solani Sorauer. Z. PflKrankh. 6: 6 (1896)

Ref.: Bordjiba et al. (2009), Bessadat et al. (2016), Bessadat et al. (2017), Ayad et al. (2017), Kedad \& Bouznad (2018), [NCBI].

Alternaria telliensis sp. nov. (Bessadat et al. 2020)

Ref.: Bessadat et al. (2020).

Alternaria tenuissima (Kunze) Wiltshire. Trans. Br. mycol. Soc. 18(2): 157 (1933)

Ref.: Dorléans \& Emmanouilidis (1972), LocquinLinard (1988), Bessadat et al. (2014), Kahoul et al. (2014), Bessadat et al. (2017), Kedad \& Bouznad (2018), Chamekh et al. (2019), [P], [NCBI].

Bipolaris oryzae (Breda de Haan) Shoemaker. Can. J. Bot. 37(5): 883 (1959)

syn. Helminthosporium macrocarpum Grev., Scott. crypt. fl. (Edinburgh) 3: 148 (1823)

Ref.: [GBIF], [P].

Bipolaris sorokiniana Shoemaker. Can. J. Bot. 37(5): 884 (1959)

Ref.: Kedad \& Bouznad (2018).

Curvularia australiensis (Bugnic. ex M.B. Ellis) Manamgoda, L. Cai \& K.D. Hyde. in Manamgoda, Cai, McKenzie, Crous, Madrid, Chukeatirote, Shivas, Tan \& Hyde, Fungal Diversity 56(1): 140 (2012)

syn. Bipolaris australiensis (Bugnic. ex M.B. Ellis) Tsuda \& Ueyama, Mycologia 73(1): 90 (1981), Drechslera australiensis Bugnic. ex M.B. Ellis, Dematiaceous Hyphomycetes (Kew): 413 (1971)

Ref.: Lunghini (1978), Chafai (1996).

Curvularia hawaiiensis (Bugnic. ex M.B. Ellis) Manamgoda. in Manamgoda, Cai, McKenzie, Crous, Madrid, Chukeatirote, Shivas, Tan \& Hyde, Fungal Diversity 56(1): 141 (2012)

syn. Drechslera hawaiiensis Bugnic. ex M.B. Ellis, Dematiaceous Hyphomycetes (Kew): 415 (1971)

Ref.: Locquin-Linard (1988).

Curvularia lunata (Wakker) Boedijn. Bull. Jard. bot. Buitenz, 3 Sér. 13(1): 127 (1933)

Ref.: Lunghini (1978), Locquin-Linard (1988).

Curvularia nicotiae (Mouch.) Y.P. Tan \& R.G. Shivas. in Tan, Madrid, Crous \& Shivas, Australas. Pl. Path. 43(6): 600 (2014) 
syn. Drechslera nicotiae Mouch., Revue Mycol., Paris 38(3-4): 108 (1975) [1974]

Ref.: CMI (1982), Manamgoda et al. (2014), [P], [GBIF], [NCBI].

Curvularia ovoidea (Hiroë) Munt.-Cvetk. Rev. agron. Noroeste Argent. 2: 322 (1957)

Ref.: Lunghini (1978).

Curvularia spicifera (Bainier) Boedijn. Bull. Jard. bot. Buitenz, 3 Sér. 13(1): 127 (1933)

syn. Drechslera spicifera (Bainier) Arx, Icon. microfung. Matsush. lect. (Kobe): 65 (1975)

Ref.: Lunghini (1978), Locquin-Linard (1988), Chamekh et al. (2019).

Curvularia subulata (Nees \& T. Nees ex Fr.) Boedijn ex J.C. Gilman. Manual of Soil Fungi: 303 (1945)

syn. Helminthosporium subulatum Nees \& T. Nees, Nova Acta Phys.-Med. Acad. Caes. Leop.-Carol. Nat. Cur. 9: 242 (1818)

Ref.: Durieu (1846), Kedad \& Bouznad (2018).

Exserohilum rostratum (Drechsler) K.J. Leonard \& Suggs. Mycologia 66(2): 290 (1974)

syn. Drechslera halodes (Drechsler) Subram. \& B.L. Jain, Curr. Sci. 35: 354 (1966)

Ref.: Lunghini (1978).

Fusicladiopsis conviva Maire. Bull. Soc. bot. Fr. 53: CLXXXVII (1907) [1906]

Ref.: Maire (1906), Saccardo (1913), Schneider-Orelli (1913), Kedad \& Bouznad (2018).

Macrosporium boucerosiae Durieu \& Mont. in Durieu, Expl. Sci. Alg., Fl. Algér. 1(livr. 9): 359 (1848) [1846-49]

Ref.: Durieu (1846), Montagne (1856), Kedad \& Bouznad (2018), [P], [GBIF].

Neoplatysporoides aloicola Crous \& M.J. Wingf. Persoonia 34: 197 (2015)

Ref.: [NCBI].

Pleospora agaves de Not. Sfer. Ital.: 73 (1863)

Ref.: Patouillard (1903), Kedad \& Bouznad (2018).

Pleospora asphodeli Rabenh. Un. ital. Crypt.: no. XII (1866)

Ref.: Jaczewski (1893), Kedad \& Bouznad (2018), [BR], [GBIF].

Pleospora bambusae Pass. Micr. ital. diagn.: no. 7 (1849)

Ref.: Saccardo \& Berlese (1886a), Kedad \& Bouznad (2018).

Pleospora chamaeropis (Durieu \& Mont.) Sacc. Syll. fung. (Abellini) 2: 262 (1883) syn. Sphaeria chamaeropis Durieu \& Mont., in Durieu, Expl. Sci. Alg., Fl. Algér. 1(livr. 13): 517 (1848) [1846-49]

Ref.: Durieu (1846), Montagne (1856), Saccardo (1883), Frohlich (1997), Kedad \& Bouznad (2018), Berlese (1892-1905).

Pleospora discors (Durieu \& Mont.) Ces. \& de Not. Comm. Soc. crittog. Ital. 1(fasc. 4): 218 (1863)

syn. Sphaeria discors Durieu \& Mont., in Durieu, Expl. Sci. Alg., Fl. Algér. 1(livr. 14): 539 (1848) [1846-49]

Ref.: Durieu (1846), Montagne (1856), Saccardo (1883), Kedad \& Bouznad (2018), [P], Berlese (18921905).

Pleospora donacina (Fr.) Niessl. Verh. nat. Ver. Brünn 14: 183 (1876)

syn. Sphaeria donacina Fr., Linnaea 5: 545 (1830)

Ref.: Durieu (1846), Kedad \& Bouznad (2018), [GBIF], [P].

Pleospora kentiae Maubl. Bull. Soc. mycol. Fr. 19(3): 291 (1903)

Ref.: Maublanc (1903), Saccardo (1905), Stevenson (1926), Mouchacca \& Saint-Jore (2000), Mouchacca (2000), Kedad \& Bouznad (2018).

Pleospora mauritanica Maire. Bull. Soc. bot. Fr. 53: CXCII (1907) [1906]

Ref.: Maire (1906), Kedad \& Bouznad (2018).

Pleospora oligomera Sacc. \& Speg. Michelia 1(no. 4): 408 (1878)

Ref.: Debeaux et al. (1880), Kedad \& Bouznad (2018).

Pleospora penicillus Fuckel. Jb. nassau. Ver. Naturk. 27-28: 23 (1874) [1873-74]

syn. Pleospora media Niessl, Verh. nat. Ver. Brünn 14: 188 (1876), Pleospora media var. limonum Penz., in Saccardo, Michelia 2(no. 8): 419 (1882)

Ref.: Saccardo \& Berlese (1886a), Kedad \& Bouznad (2018), Berlese (1892-1905).

Pleospora rhanterii Henn. Annuaire Conser. et Jard. bot. Genève 7: 242 (1904)

Ref.: Hennings (1902), Kedad \& Bouznad (2018).

Pleospora solorinae (Mont.) Sacc. Syll. fung. (Abellini) 2: 274 (1883)

syn. Sphaeria solorinae Mont., Annls Sci. Nat., Bot., sér. 4 8: 307 (1857)

Ref.: Montagne (1857).

Pyrenophora graminea S. Ito \& Kurib. Proc. Imp. Acad. Japan 6(8): 353 (1930)

Ref.: Kedad \& Bouznad (2018). 
Pyrenophora teres Drechsler. J. Agric. Res., Washington 24(8): 656 (1923)

Ref.: Kedad \& Bouznad (2018).

Pyrenophora tritici-repentis (Died.) Drechsler. J. Agric. Res., Washington 24(8): 667 (1923)

Ref.: Benslimane et al. (2011), Kedad \& Bouznad (2018).

Stemphylium dendriticum Sousa da Câmara. Bol. Acad. Ci. Lisboa: 12 [repr.] (1930)

syn. Alternaria dendritica (Sousa da Câmara) P. Joly, Le Genre Alternaria (Encycl. Mycol.): 147 (1964))

Ref.: [P], [VKM].

Stemphylium lycopersici (Enjoji) W. Yamam. Trans. Mycol. Soc. Japan 2(no. 5): 93 (1960)

Ref.: Kedad \& Bouznad (2018), [NCBI].

Stemphylium solani G.F. Weber. Phytopathology 20: 516 (1930)

Ref.: Lakhdari (2017), Kedad \& Bouznad (2018).

Stemphylium verruculosum (O.E.R. Zimm.) Sacc. Syll. fung. (Abellini) 4: 522 (1886)

Ref.: Dubost (1966).

Stemphylium vesicarium (Wallr.) E.G. Simmons. Mycologia 61(1): 9 (1969)

syn. Macrosporium commune Rabenh., Fungi europ. exsicc.: no. 1360 (1870), Pleospora excavata var. basitricha (Durieu \& Mont.) Sacc., Syll. fung. (Abellini) 2: 248 (1883), Pleospora herbarum (Pers.) Rabenh., Klotzschii Herb. Viv. Mycol., Edn Nov, Ser. Sec., Cent. 6: no. 547 (1857), Sphaeria excavata var. basitricha Durieu \& Mont., in Durieu, Expl. Sci. Alg., Fl. Algér. 1(livr. 14): 526 (1848) [1846-49], Sphaeria herbarum Pers., Syn. meth. fung. (Göttingen) 1: 78 (1801), Sphaeria pisi Sowerby, Col. fig. Engl. Fung. Mushr. (London) 3(no. 27): tab. 393, fig. 8 (1803))

Ref.: Durieu (1846), Montagne (1856), Saccardo \& Roumeguère (1881, 1885), Saccardo (1883), Saccardo \& Berlese (1886a), Berlese (1892-1905), Maire (1906), Killian \& Fehér (1935), Dubost (1966), Kedad \& Bouznad (2018), [BR], [GBIF], [P].

\section{Polystomellaceae}

Dothidella oleandrina (Durieu \& Mont.) Sacc. Syll. fung. (Abellini) 2: 632 (1883)

syn. Dothidea oleandrina Durieu \& Mont., in Durieu, Expl. Sci. Alg., Fl. Algér. 1(livr. 14): 547 (1848) [1846-49], Didymella oleandri (Durieu \& Mont.) Sacc. Syll. fung. (Abellini) 1: 551 (1882), , Sphaeria oleandri Durieu \& Mont., in Durieu 1848

Ref.: Durieu (1846), Montagne (1856), Saccardo (1982b, 1883), Patouillard (1903), Kedad \& Bouznad
(2018), [GBIF], [P], [S], [SVH], Biodiv. Atlas Swed., [GBIF].

Dothidella ulmi (C.-J. Duval) G. Winter. Rabenh. Krypt.-Fl., Edn 2 (Leipzig) 1.2: 904 (1886)

syn. Dothidea ulmi (C.-J. Duval) Fr., Syst. mycol. (Lundae) 2(2): 555 (1823), Phyllachora ulmi (C.-J. Duval) Fuckel, Jb. nassau. Ver. Naturk. 23-24: 218 (1870) [1869-70], Systremma ulmi (J. Schröt.) Theiss. \& Syd., Annls mycol. 13(3/4): 334 (1915)

Ref.: Durieu (1846), Patouillard (1903), Stevenson (1926), Kedad \& Bouznad (2018), [GBIF], [P].

\section{Pseudoperisporiaceae}

Dimerina cyanophila Feldmann. Bull. Soc. Hist. nat. Afr. N. 35: 14 (1944)

Ref.: Feldmann (1944), Frohlich (1997).

\section{Saccotheciaceae}

Aureobasidium pullulans (de Bary \& Löwenthal) G. Arnaud. Annals d'École National d'Agric. de Montpellier, Série 2 16(1-4): 39 (1918) [1917]

Ref.: Nicot (1955), Dorléans \& Emmanouilidis (1972), Harzallah et al. (2009), Bensaci et al. (2015), [P].

Metasphaeria algeriensis Sacc. \& Berl. Revue mycol., Toulouse 8(no. 29): 34 (1886)

Ref.: Saccardo \& Berlese (1886a), Saccardo (1886a, 1891), Aptroot (1998), Kedad \& Bouznad (2018).

Metasphaeria anisometra (Cooke \& Harkn.) Sacc. Syll. fung. (Abellini) 2: 163 (1883)

Ref.: Berlese (1892-1905, 1894).

Metasphaeria calamina (Durieu \& Mont.) Sacc. Syll. fung. (Abellini) 2: 178 (1883)

syn. Sphaeria calamina Durieu \& Mont., in Durieu, Expl. Sci. Alg., Fl. Algér. 1(livr. 14): 532 (1848) [1846-49]

Ref.: Durieu (1846), Montagne (1856), Saccardo (1883), Saccardo \& Berlese (1886a), Berlese (18921905), Berlese (1894), Patouillard (1903), Kedad \& Bouznad (2018), [GBIF], [P].

Metasphaeria debeauxii (Sacc. \& Roum.) Sacc. Syll. fung. (Abellini) 2: 182 (1883)

Ref.: Saccardo (1883), Berlese (1894), Frohlich (1997), Berlese (1892-1905), [USDA].

Metasphaeria ferulina (Durieu \& Mont.) Sacc. Syll. fung. (Abellini) 2: 160 (1883)

syn. Sphaeria ferulina Durieu \& Mont., in Durieu, Expl. Sci. Alg., Fl. Algér. 1(livr. 13): 484 (1848) [1846-49]

Ref.: Durieu (1846), Saccardo (1883), Berlese (18921905, 1894), Kedad \& Bouznad (2018), [GBIF], [P]. 
Metasphaeria hederae (Sowerby) Sacc. Syll. fung. (Abellini) 2: 169 (1883)

syn. Sphaeropsis leucostigma DC., Fl. franç., Edn 3 (Paris) 5/6: 144 (1815)

Durieu (1846), Kedad \& Bouznad (2018).

Metasphaeria inulina (Durieu \& Mont.) Sacc. Syll. fung. (Abellini) 2: 162 (1883)

syn. Sphaeria inulina Durieu \& Mont., in Durieu, Expl. Sci. Alg., Fl. Algér. 1(livr. 14): 529 (1848) [1846-49]

Ref.: Durieu (1846), Montagne (1856), Saccardo (1883), Berlese (1894), Kedad \& Bouznad (2018), [P], [GBIF], Berlese (1892-1905).

Metasphaeria iridicola Sacc. Syll. fung. (Abellini) 2: 178 (1883)

syn. Sphaeria iridis Durieu \& Mont., in Durieu, Expl. Sci. Alg., Fl. Algér. 1(livr. 14): 540 (1848) [1846-49]

Ref.: Durieu (1846), Montagne (1856), Saccardo (1883), Stevenson (1926), Kedad \& Bouznad (2018), [P], [GBIF], Berlese (1892-1905).

Metasphaeria scalaris (Durieu \& Mont.) Sacc. Syll. fung. (Abellini) 2: 168 (1883)

syn. Sphaeria scalaris Durieu \& Mont., in Durieu, Expl. Sci. Alg., Fl. Algér. 1(livr. 13): 510 (1848) [1846-49]

Ref.: Durieu (1846), Montagne (1856), Saccardo (1883), Berlese (1892-1905), Kedad \& Bouznad (2018)[P], [GBIF],

Metasphaeria scotophila (Durieu \& Mont.) Sacc. Syll. fung. (Abellini) 2: 160 (1883)

syn. Sphaeria scotophila Durieu \& Mont., in Durieu, Expl. Sci. Alg., Fl. Algér. 1(livr. 14): 531 (1848) [1846-49]

Ref.: Durieu (1846), Montagne (1856), Saccardo (1883), Kedad \& Bouznad (2018).

Pseudoseptoria obscura Quaedvl., Verkley \& Crous. Stud. Mycol. 75: 361 (2013)

Ref.: [NCBI].

Saccothecium sepincola (Fr.) Fr. Summa veg. Scand., Sectio Post. (Stockholm): 398 (1849)

syn. Phacidium sepincola (Fr.) Fr., K. svenska Vetensk-Akad. Handl., ser. 3 40: 107 (1819), Sphaerulina sepincola (Fr.) Starbäck, Bot. Notiser: 117 (1890)

Ref.: Durieu (1846), Saccardo (1883), Kedad \& Bouznad (2018), [GBIF], [P].

Selenophoma catananches Maire. Bull. Soc. bot. Fr. 53: CLXXXVII (1907) [1906]

Ref.: Maire (1906), Kedad \& Bouznad (2018).
Selenophoma superflua (Sacc.) Dejeva. in Byzova et al., Flora Sporovykh Rasteniǐ Kazakhstana [Cryptogamic Flora of Kazakhstan], 5, Nesovershennye Griby - Fungi Imperfecti (Deuteromycetes) 1. Sphaeropsidales (Alma-Ata): 300 (1967)

syn. Phoma superflua Sacc., Michelia 1(no. 5): 522 (1879)

Ref.: Saccardo \& Berlese (1886a), Kedad \& Bouznad (2018).

\section{Sporormiaceae}

Chaetopreussia chadefaudii Locq.-Lin. Revue Mycol., Paris 41(2): 187 (1977)

Ref.: Locquin-Linard (1977b), Locquin-Linard (1988).

Preussia commutata (Niessl) Abdullah \& Guarro. in Abdullah, Al-Saadoon \& Guarro, Nova Hedwigia 69(1-2): 214 (1999)

syn. Sporormiella commutata (Niessl) S.I. Ahmed \& Cain, Can. J. Bot. 50(3): 437 (1972)

Ref.: Locquin-Linard (1988).

Preussia dubia (S.I. Ahmed \& Cain) Kruys. in Kruys \& Wedin, Syst. Biodiv. 7(4): 476 (2009)

syn. Sporormiella dubia S.I. Ahmed \& Cain, Can. J. Bot. 50(3): 440 (1972)

Ref.: Locquin-Linard (1988).

Preussia typharum (Sacc.) Cain. Can. J. Bot. 39: 1638 (1961)

Ref.: Locquin-Linard (1988).

Preussia vulgaris (Corda) Cain. Can. J. Bot. 39: 1642 (1961)

syn. Perisporium vulgare Corda, Icon. fung. (Prague) 2: fig. 97 (1838)

Ref.: Saccardo \& Berlese (1886a), Faurel \& Schotter (1964), Kedad \& Bouznad (2018).

Sporormia deserticola Faurel \& Schotter. Revue Mycol., Paris 29(4): 291 (1964)

Ref.: Faurel \& Schotter (1964), CMI (1968).

Sporormia mirabilis Breton \& Faurel. Bull. trimest. Soc. mycol. Fr. 80: 254 (1964)

Ref.: CMI (1968).

Sporormiella australis (Speg.) S.I. Ahmed \& Cain. Can. J. Bot. 50(3): 434 (1972)

Ref.: Locquin-Linard (1988).

Sporormiella capybarae (Speg.) S.I. Ahmed \& Cain. Can. J. Bot. 50(3): 436 (1972)

Ref.: Locquin-Linard (1988). 
Sporormiella dakotensis (Griffiths) S.I. Ahmed \& Cain. Can. J. Bot. 50(3): 439 (1972)

Ref.: Locquin-Linard (1988).

Sporormiella intermedia (Auersw.) S.I. Ahmed \& Cain ex Kobayasi. in Kobayasi, Hiratsuka, Otani, Tubaki, Udagawa \& Soneda, Bull. natn. Sci. Mus., Tokyo 12: 339 (1969)

Ref.: Faurel \& Schotter (1964), Locquin-Linard (1988).

Sporormiella isomera S.I. Ahmed \& Cain. Can. J. Bot. 50(3): 445 (1972)

Ref.: Locquin-Linard (1988).

Sporormiella lageniformis (Fuckel) S.I. Ahmed \& Cain. Can. J. Bot. 50(3): 446 (1972)

syn. Sporormia ambigua Niessl, Oesterr. bot. Z. 28: 97 (1878)

Ref.: Faurel \& Schotter (1964).

Sporormiella megalospora (Auersw.) S.I. Ahmed \& Cain Can. J. Bot. 50(3): 449 (1972)

Ref.: Locquin-Linard (1988).

Sporormiella minima (Auersw.) S.I. Ahmed \& Cain. in Ahmed \& Asad, Pakist. J. scient. ind. Res. 12(3): 241 (1970)

syn. Sporormiopsis minima (Auersw.) Breton \& Faurel, Bull. trimest. Soc. mycol. Fr. 80: 257 (1964)

Ref.: Breton \& Faurel (1964), Locquin-Linard (1988).

Sporormiella ovina (Desm.) S.I. Ahmed \& Cain. Can. J. Bot. 50(3): 454 (1972)

syn. Sporormia gigantea E.C. Hansen, Vidensk. Meddel. Dansk Naturhist. Foren. Kjøbenhavn 59: 113 (1877) [1876]

Ref.: Faurel \& Schotter (1964).

Sporormiella pulchella (E.C. Hansen) S.I. Ahmed \& Cain. Can. J. Bot. 50(3): 456 (1972)

syn. Sporormia pulchella E.C. Hansen, Vidensk. Meddel. Dansk Naturhist. Foren. Kjøbenhavn 59: 114 (1877) [1876]

Ref.: Faurel \& Schotter (1964), Faurel \& Schotter (1964), Locquin-Linard (1988).

Sporormiella similis R.S. Khan \& Cain. Can. J. Bot. 57(10): 1178 (1979)

syn. Preussia similis (R.S. Khan \& Cain) Arenal, in Arenal, Platas \& Peláez, Mycotaxon 89(1): 140 (2004) Noumeur et al. (2017), [NCBI].

Sporormiella subtilis S.I. Ahmed \& Cain. Can. J. Bot. 50(3): 459 (1972)

Ref.: Locquin-Linard (1988).
Sporormiella teretispora S.I. Ahmed \& Cain. Can. J. Bot. 50(3): 460 (1972)

Ref.: Locquin-Linard (1988).

Sporormiella vexans (Auersw.) S.I. Ahmed \& Cain. Can. J. Bot. 50(3): 374 (1972)

Ref.: Faurel \& Schotter (1964), Locquin-Linard (1988).

Westerdykella multispora (Saito \& Minoura ex Cain) Cejp \& Milko. Česká Mykol. 18(2): 84 (1964)

Ref.: [P].

Westerdykella nigra (Routien) Arx. Proc. K. Ned. Akad. Wet., Ser. C, Biol. Med. Sci. 76(3): 294 (1973)

syn. Preussiella nigra (Routien) Lodha, in Subramanian (Ed.), Taxonomy of Fungi (Proc. int. Symp. Madras, 1973), Pt 1: 251 (1978)

Ref.: Locquin-Linard (1988).

\section{Strigulaceae}

Oletheriostrigula papulosa (Durieu \& Mont.) Huhndorf \& R.C. Harris. Brittonia 48(4): 551 (1996) syn. Leptosphaeria papulosa (Durieu \& Mont.) Sacc., Nuovo G. bot. ital. 7(4): 313 (1875), Massarina papulosa (Durieu \& Mont.) S.K. Bose, Phytopath. Z. 41: 176 (1961), Metasphaeria papulosa (Durieu \& Mont.) Sacc., Syll. fung. (Abellini) 2: 168 (1883), Sphaeria papulosa Durieu \& Mont., Expl. Sci. Alg., Fl. Algér. 1(livr. 14): 536 (1848) [1846-49]

Ref.: Durieu (1846), Montagne (1856), Saccardo (1882a, 1883), Aptroot (1998), Kedad \& Bouznad (2018), [GBIF], [P].

\section{Teichosporaceae}

Teichospora brevirostris (Fr.) Fuckel. Jb. nassau. Ver. Naturk. 23-24: 161 (1870) [1869-70]

Ref.: [MPU], [GBIF].

Teichospora sarmenticia Sacc. \& Speg. Michelia 1(no. 3): 349 (1878)

Ref.: Saccardo \& Roumeguère (1881), Saccardo (1883), Kedad \& Bouznad (2018).

Teichospora brachyasca Ellis \& Everh. Bull. Torrey bot. Club 27(2): 52 (1900)

Ref.: Berlese (1892-1905).

\section{Teratosphaeriaceae}

Teratosphaeria molleriana (Thüm.) Crous \& U. Braun, in Crous, Braun \& Groenewald, Stud. Mycol. 58: 10 (2007)

syn. Mycosphaerella molleriana (Thüm.) Lindau, in Engler \& Prantl, Nat. Pflanzenfam., Teil. I (Leipzig) 1(1): 424 (1897), Sphaerella molleriana Thüm., Inst. 
Coimbra 28: 31 (1880)

Ref.: Roumeguère (1887b), Stevenson (1926), Stevenson (1926).

\section{Testudinaceae}

Lepidosphaeria nicotiae Parg.-Leduc. Pubbl. Staz. Zool. Napoli, I 270: 2786 (1970)

Ref.: CMI (1982), [GBIF], [NCBI].

Pseudophaeotrichum sudanense Arx, E. Müll. \& C. Stoll. Nova Hedwigia 17: 84 (1969)

Ref.: Locquin-Linard (1988).

\section{Torulaceae}

Dendryphion nodulosum Sacc. Michelia 1(no. 1): 81 (1877)

Ref.: Faurel \& Schotter (1964).

Torula herbarum (Pers.) Link. Mag. Gesell. naturf. Freunde, Berlin 3(1-2): 19 (1809)

Ref.: Durieu (1846), Saccardo \& Roumeguère (1881), Saccardo \& Berlese (1886a), Saccardo (1886b), Locquin-Linard (1988), Kedad \& Bouznad (2018).

Torula morrhuae (Farl.) Sacc. in Trotter, Syll. fung. (Abellini) 25: 762 (1931)

Ref.: Saccardo (1931).

\section{Trematosphaeriaceae}

Trematosphaeria pertusa Fuckel. Jb. nassau. Ver. Naturk. 23-24: 161 (1870) [1869-70]

syn. Sphaeria pertusa Pers., Syn. meth. fung. (Göttingen) 1: 83 (1801)

Ref.: Durieu (1846), Kedad \& Bouznad (2018), [GBIF], [P].

Trematosphaeria pyrenogena (Durieu \& Mont.) Sacc. Syll. fung. (Abellini) 2: 121 (1883)

syn. Sphaeria pyrenogena Durieu \& Mont., in Durieu, Expl. Sci. Alg., Fl. Algér. 1(livr. 13): 501 (1848) [1846-49]

Ref.: Durieu (1846), Montagne (1856), Saccardo (1883), Berlese (1892-1905, Kedad \& Bouznad (2018), [GBIF], [P].

\section{Valsariaceae}

Myrmaecium rubricosum (Fr.) Fuckel, Jb. nassau. Ver. Naturk. 23-24: 227 (1870) [1869-70]

syn. Hypoxylon rubricosum (Fr.) Fr., Summa veg. Scand., Sectio Post. (Stockholm): 384 (1849), Sphaeria rubricosa Fr., Elench. fung. (Greifswald) 2: 63 (1828), Valsaria rubricosa (Fr.) Sacc., Syll. fung. (Abellini) 1: 743 (1882)

Ref.: Durieu (1846), Kedad \& Bouznad (2018), [P].
Valsaria insitiva (Tode) Ces. \& de Not. Comm. Soc. crittog. Ital. 1(fasc. 4): 205 (1863)

syn. Sphaeria notarisii Durieu \& Mont., in Durieu, Expl. Sci. Alg., Fl. Algér. 1(livr. 12): 470 (1848) [1846-49], Sphaeria phlyctaenodes Mont., in Durieu, Expl. Sci. Alg., Fl. Algér. 1: 468 (1849) [1846-49], Sphaeria viticola Schwein., Schr. naturf. Ges. Leipzig 1: 34 [8 of repr.] (1822), Valsaria notarisii (Durieu \& Mont.) Sacc., Syll. fung. (Abellini) 1: 742 (1882), Valsaria phlyctaenodes (Mont.) Sacc., Syll. fung. (Abellini) 1: 747 (1882), , Valsa notarisii (Durieu \& Mont.) Mont., Syll. gen. sp. crypt. (Paris): 218 (1856), Valsa phlyctaenodes (Mont.) Mont., Syll. gen. sp. crypt. (Paris): 221 (1856)

Ref.: Durieu (1846), Montagne (1856), Saccardo (1882b), Kedad \& Bouznad (2018), [GBIF], [P].

\section{Venturiaceae}

Fusicladium pyracanthae (Thüm.) O. Rostr. Hedwigia 52: 270 (1912)

Ref.: Kedad \& Bouznad (2018).

Lasiobotrys hispanicus Theiss. \& Syd. Annls mycol. 16(1/2): 177 (1918)

Ref.: Maire \& Werner (1937), Kedad \& Bouznad (2018), [USDA].

Lasiobotrys lonicerae (Fr.) Kunze. in Kunze \& Schmidt, Mykologische Hefte (Leipzig) 2: 88 (1823)

Ref.: Durieu (1846), Saccardo (1882b), Battandier et al. (1914), Maire (1915c), Kedad \& Bouznad (2018), [MA], [BR], [P], [CABI], [GBIF], [USDA].

Venturia inaequalis (Cooke) G. Winter. in Thümen, Mycoth. Univ., cent. 3: no. 261 (1875)

Ref.: Kedad \& Bouznad (2018).

Venturia oleaginea (Castagne) Rossman \& Crous in Rossman, Crous \& Hyde, IMA Fungus 6(2): 520 (2015)

syn. Cycloconium oleagineum Castagne [as 'oleaginum'], Cat. Pl. Mars.: 220 (1845), Fusicladium oleagineum (Castagne) Ritschel \& U. Braun, in Schubert, Ritschel \& Braun, Schlechtendalia 9: 70 (2003)

Ref.: Roumeguère (1887b), Patouillard (1903), Maire (1919), Guechi \& Girre (1994), Schubert et al. (2003), Chliyeh et al. (2014), Kedad \& Bouznad (2018), [MA], [BR], [GBIF], [USDA].

Venturia pyrina Aderh. Landwirtschaftliche Jahrbucher 25: 875 (1896)

Ref.: Kedad \& Bouznad (2018).

Venturia rumicis (Desm.) G. Winter. Rabenh. Krypt.Fl., Edn 2 (Leipzig) 1.2: 435 (1885) 
Maire (1917a), Kedad \& Bouznad (2018), [MPU], [GBIF].

\section{Zopfiaceae}

Zopfia rhizophila Rabenh. Fungi europ. exsicc., Cent. 13: no. 44 (1874)

Patouillard (1903), Kedad \& Bouznad (2018).

\section{Dothideomycetes with uncertain position (Incertae sedis)}

Asteromella bupleuri (Fuckel) Aa. in van der Aa \& Vanev, A Revision of the Species Described in Phyllosticta (Utrecht): 116 (2002)

syn. Depazea bupleuri Fuckel, Hedwigia 3: 156 (1864), Phyllosticta bupleuri (Fuckel) Sacc., Syll. fung. (Abellini) 3: 46 (1884)

Saccardo (1884), Roumeguère (1885), Stevenson (1926), Kedad \& Bouznad (2018), [BR], [S], [SVH], [GBIF].

Berkleasmium granulosum (Durieu \& Mont.) R.T. Moore. Mycologia 50(5): 687 (1959) [1958]

syn. Sporidesmium granulosum Durieu \& Mont., in Durieu, Expl. Sci. Alg., Fl. Algér. 1(livr. 9): 330 (1848) [1846-49]

Ref.: Durieu (1846), Montagne (1856), Kedad \& Bouznad (2018).

Camarosporium aequivocum (Pass.) Sacc. Syll. fung. (Abellini) 3: 467 (1884)

Ref.: Roumeguère (1885), [MIN], [BR], [GBIF].

Camarosporium dalmaticum (Thüm.) Zachos \& Tzav.-Klon. Annls Inst. phytopath. Benaki, N.S. 12(1): 69 (1979)

Ref.: Kedad \& Bouznad (2018).

Camarosporium guyonianum (Mont. \& Durieu) Van Warmelo \& B. Sutton. Mycol. Pap. 145: 26 (1981) syn. Didymosporium guyonianum Mont. \& Durieu, in Durieu, Expl. Sci. Alg., Fl. Algér. 1(livr. 9): 330 (1848) [1846-49], Stegonsporium guyonianum (Mont. $\&$ Durieu) Sacc., Syll. fung. (Abellini) 3: 806 (1884)

Ref.: Durieu (1846), Montagne (1856), Saccardo (1884), Kedad \& Bouznad (2018), [GBIF], [P].

Camarosporium minutulum (Durieu \& Lév.) Sacc. Syll. fung. (Abellini) 3: 469 (1884)

Ref.: Saccardo (1884), Kedad \& Bouznad (2018).

Camarosporium oreades (Durieu \& Mont.) Sacc. Syll. fung. (Abellini) 3: 466 (1884)

syn. Hendersonia oreades Durieu \& Mont., in Durieu, Expl. Sci. Alg., Fl. Algér. 1(livr. 15): 571 (1849) [1846-49]
Ref.: Durieu (1846), Saccardo (1884), Kedad \& Bouznad (2018).

Camarosporium retamae Trab. ex Sacc. Syll. fung. (Abellini) 10: 339 (1892)

Ref.: Saccardo (1892), Kedad \& Bouznad (2018).

Camarosporium roumeguerei var. halimi Maire. Bull. Soc. bot. Fr. 53: CLXXXIX (1907) [1906]

Ref.: Maire (1906), Kedad \& Bouznad (2018).

Catinella olivacea (Batsch) Boud. Hist. Class. Discom. Eur. (Paris): 150 (1907)

Ref.: [MPU], [GBIF].

Endococcus rugulosus Nyl. Mém. Soc. Imp. Sci. Nat. Cherbourg 3: 193 (1855)

syn. Discothecium gemmiferum var. calcaricola (Mudd) Keissl. [as 'calcaricolum'], Rabenh. Krypt.-Fl., Edn 2 (Leipzig) 8: 389 (1930)

Ref.: Vouaux (1912), Maire \& Werner (1937).

Homostegia asparagi (Fuckel) Höhn. Mitt. bot. Inst. tech. Hochsch. Wien 4(2): 43 (1927)

syn. Diaporthe asparagi Fuckel, Jb. nassau. Ver. Naturk. 23-24: 206 (1870) [1869-70]

Ref.: [MPU], [GBIF].

Hyalosphaera pulchella (F. Stevens) Rossman. Mycol. Pap. 157: 56 (1987)

syn. Dexteria pulchella F. Stevens, Trans. Ill. St. Acad. Sci. 10: 174 (1917) Macrosporium atrum Bull. Soc. mycol. Fr. 29: 456 (1913)

Ref.: Vouaux (1912), Saccardo (1928).

Hysterographium fraxini (Pers.) De Not., G. bot. ital. 2(2): 22 (1847)

syn. Hysterium confluens Wallr., Fl. crypt. Germ. (Norimbergae) 2: 440 (1833), H. elongatum Wahlenb., Fl. lapp.: 528 (1812), H. fraxini Pers., Neues Mag. Bot. 1: 85 (1794), Hysterographium elongatum (Wahlenb.) Corda, Icon. fung. (Prague) 5: 77 (1842)

Ref.: Durieu (1846), Kedad \& Bouznad (2018), [MPU], [P], [CABI], [GBIF], [GBIF], [GBIF].

Leptospora rubella (Pers.) Rabenh. Klotzschii Herb. Viv. Mycol., Edn Nov, Ser. Sec., Cent. 6: no. 532 (1857)

syn. Sphaeria rubella Pers., Syn. meth. fung. (Göttingen) 1: 63 (1801)

Ref.: Durieu (1846), Kedad \& Bouznad (2018).

Leptothyrium holoschoeni (Durieu \& Mont.) Sacc. Syll. fung. (Abellini) 3: 636 (1884)

Ref.: Saccardo (1884), Kedad \& Bouznad (2018).

Leptothyrium rhodomelas Maire. Bull. Soc. Hist. nat. Afr. N. 8(7): 182 (1917) 
Ref.: Maire (1917a), Kedad \& Bouznad (2018), [MPU], [GBIF].

Neopeckia oryzopsis Maire

Ref.: Maire (1933), Kedad \& Bouznad (2018).

Periconia algeriana Lunghini. G. bot. ital., n.s. 112(56): 383 (1978) [1979]

Ref.: Lunghini (1978), CMI (1982), [GBIF].

Periconia atra Corda. Icon. fung. (Prague) 1: 19 (1837) Icon. fung. (Prague) 1: 19 (1837)

Ref.: Killian \& Fehér (1935).

Periconia byssoides Pers. Syn. meth. fung. (Göttingen) 2: 686 (1801)

syn. Periconia pycnospora Fresen., Beitr. Mykol. 1: 20 (1850)

Ref.: Durieu (1846), Maire (1919), Maire \& Werner (1937), Kedad \& Bouznad (2018), [MA], [GBIF], [USDA].

Periconia genistae Lunghini. G. bot. ital., n.s. 112(56): 387 (1978) [1979]

Ref.: Lunghini (1978), [GBIF].

Periconia macrospinosa Lefebvre \& Aar.G. Johnson. Mycologia 41(4): 417 (1949)

Ref.: Kahoul et al. (2014).

Periconia sahariana Lunghini. G. bot. ital., n.s. 112(56): 390 (1978) [1979]

Ref.: Lunghini (1978), CMI (1982), [GBIF].

Macrosporium atrum Mycol. 75: 22 (2012) [2013]

syn. Deuterophoma tracheiphila Petri, Boll. R. Staz. Patalog. Veget. Roma 9: 396 (1929)

Ref.: [CABI].

Pyrenochaeta hepaticarum Sacc. \& Trotter. Syll. fung. (Abellini) 22(2): 934 (1913)

Ref.: Saccardo (1913).

Rhopographus filicinus (Fr.) Nitschke ex Fuckel. Jb. nassau. Ver. Naturk. 23-24: 219 (1870) [1869-70]

syn. Sphaeria filicina Fr., Syst. mycol. (Lundae) 2(2): 427 (1823)

Ref.: Durieu (1846), Kedad \& Bouznad (2018), [GBIF], [P].

Remotididymella destructiva (Plowr.) Valenz.-Lopez, Cano, Crous, Guarro \& Stchigel. in Valenzuela-Lopez, Cano-Lira, Guarro, Sutton, Wiederhold, Crous \& Stchigel, Stud. Mycol. 90: 36 (2017)

syn. Phoma destructiva Plowr., Gard. Chron., N.S. 16: 621 (1881)

Ref.: Kedad \& Bouznad (2018).
Scolecostigmina palmivora (Sacc.) Kamal. Cercosporoid Fungi of India (Dehra Dun): 260 (2010)

Ref.: Kedad \& Bouznad (2018).

Septoriella vagans (Niessl) Y. Marín \& Crous, in Marin-Felix, Hernández-Restrepo, Iturrieta-González, García, Gené, Groenewald, Cai, Chen, Quaedvlieg, Schumacher, Taylor, Ambers, Bonthond, Edwards, Krueger-Hadfield, Luangsa-ard, Morton \& Moslemi, Stud. Mycol. 94: 111 (2019)

syn. Leptosphaeria praeclara f. typhiseda (Sacc. \& Berl.) Berl., Icon. fung. (Abellini) 1(2): 75 (1892), Leptosphaeria typhiseda Sacc. \& Berl., Revue mycol., Toulouse 8(no. 29): 33 (1886)

Ref.: Saccardo \& Berlese (1886a), Saccardo (1886a, 1891), Berlese (1892-1905, 1894), Kedad \& Bouznad (2018), [ILL NY Herbarium ], [CABI].

Sporidesmium folliculatum (Corda) E.W. Mason \& S. Hughes. in Hughes, Can. J. Bot. 31(5): 609 (1953)

syn. Helminthosporium folliculatum Corda [as 'Helmisporium'], Icon. fung. (Prague) 1: 12 (1837)

Ref.: Killian \& Fehér (1935).

Stuartella formosa Fabre. Annls Sci. Nat., Bot., sér. 6 9: 95 (1879) [1878]

Ref.: [USDA].

Thalassoascus tregoubovii Ollivier. C. r. hebd. Séanc. Acad. Sci., Paris 182: 1348 (1926)

syn. Melanopsamma tregoubovii (Ollivier) Ollivier,

Ann. Inst. océanogr. Monaco 7: 167 (1930)

Ref.: Feldmann (1932), Kohlmeyer (1963).

Zymoseptoria tritici (Roberge ex Desm.) Quaedvl. \& Crous, in Quaedvlieg, Kema, Groenewald, Verkley, Seifbarghi, Razavi, Mirzadi Gohari, Mehrabi \& Crous, Persoonia 26: 67 (2011)

syn. Mycosphaerella graminicola (Fuckel) J. Schröt., in Cohn, Krypt.-Fl. Schlesien (Breslau) 3.2(3): 340 (1894) [1908], Septoria tritici Roberge ex Desm., Annls Sci. Nat., Bot., sér. 2 17: 107 (1842)

Ref.: Benbelkacem et al. (2011), Allioui et al. (2016), Kedad \& Bouznad (2018), [CABI], [CABI], [GBIF], [NCBI].

\section{Doubtful records unsupported by literature data}

Ascospora debeauxii - Collector: Debeaux 1881 [SVH].

Cercospora lamarckiae, noted sp. nov. - Collector: Maire 1918 - [MPU].

Depazea withaniae - Collector: Debeaux. 1880 [BR]. 
Didymella maura Maire - Collector: Duvernoy 1919 [MPU].

Didymella rutae Maire - Collector: Duvernoy 1919 [MPU].

Diplodia mammaeformis Lév. - [P].

Hysterium duplicatum Durieu \& Mont. - [P].

Lophiostoma helichrysi var. artemisiae Maire - (syn.) - [MPU].

Macrosporium atrum Durieu \& Mont. Fungi europ. exsicc. Klotzschii herbarii vivi mycologici continuatio, Edn nova. Series secunda, Cent. 3: no. 273 (1860) Collector Montagne ? - [P].

Microdiplodia oreopanacis - Collector: Duvernoy 1919 - [MPU].

Phleospora ferulae - Collector: Duvernoy 1919 [MPU].

Pleospora daucina Sacc. - Saccardo (1883).

Phoma oreopanacis - Collector: Duvernoy 1919 [MPU].

Rhopographus asparagi - noted sp. nov. - Collector: Duvernoy 1919 - [MPU].

\section{Conclusions}

During our extensive survey, 562 species belonging to 59 families and 176 genera were recorded. Therefore, it should be mentioned here that, although the present study will add some new data to our information concerning Fungi in Algeria, this checklist must be considered as a provisional one always waiting for continuous supplementation.

\section{References}

Abdel-Azeem AM, Salem FM 2013. A checklist of Egyptian fungi: I. Protozoan fungal analogues. Mycosphere 4(4), 794-807.

Abdel-Azeem AM, Abu-Elsaoud A, Darwish A, Balbool B, Abo Nouh F, Abo Nahas H, Abd El-Azeem M, Ali N, Kirk P 2020. The Egyptian Ascomycota 1: Genus Aspergillus.Microbial Biosystems, 5(1), pp. 61-99. doi: 10.21608/mb.2020.100044.

Abdelaziz O, Senoussı MM, Oufroukh A, Birgücü AK, Karaca İ 2018. evaluation of different isolates of entomopathogenic fungi against Metopolophium dirhodum (Walker) (Homoptera: Aphididea) from
Constantine, Algeria. International Journal of Innovative Approaches in Agricultural Research, 2(1): 35-43, DOI:10.29329/ijiaar.2018.133.4.

Allioui N, Siah A, Brinis L, Reignault P, Halama P 2016. Identiication of QoI fungicide-resistant genotypes of the wheat pathogen Zymoseptoria tritici in Algeria. Phytopathologia Mediterranea, 55(1): 89-97. DOI: 10.14601/Phytopathol_Mediterr-16235.

Amrani S, Abdel-Azeem AM 2018a. Check-list of Algerian fungi - Part 1: Protozoan fungal analogues (Myxomycetes). Microbial Biosystems 3(1):45-60. (Peer reviewed).

Amrani S, Abdel-Azeem AM 2018b. Check-list of Algerian fungi - Part 2: Chromistan Fungal Analogues (Oomycota, Bigyra, Cercozoa). Microbial Biosystems 3(2):13-28. (Peer reviewed).

Amrani S, Abdel-Azeem AM 2019. Check-list of Algerian fungi - Part 3: Laboulbeniales (Ascomycota). Microbial Biosystems 4(1):17-30. (Peer reviewed)

Amrani S, Abo Nahas H, Ata T, Abdel-Azeem AM 2019. Checklist of Algerian fungi - Part 4: Sordariomycetes (Ascomycota)', Microbial Biosystems, 4(3), pp. 14-54. doi: 10.21608/mb.2019.65799. (Peer reviewed)

Andreánszky G 1934. Plantae in Africa boreali lectae I. Index horti botanici Universitatis Budapestinensis, 2: 67-110.

Aptroot A1995. A monograph of Didymosphaeria. Studies in mycology, 37: 1-160.

Aptroot A 1998. A world revision of Massarina (Ascomycota). Nova Hedwigia 66(1-2): 89-162.

Ayad D, Leclerc S, Hamon B, Kedad A, Bouznad Z, Simoneau P 2017. First report of early blight caused by Alternaria protenta on potato in Algeria. Plant Disease, 101(5): 836-837, DOI: 10.1094/PDIS-10-16-1420-PDN. (5), pp.836-837.

Ayad D, Aribi D, Hamon B, Kedad A, Simoneau P, Bouznad Z 2018. New data on early blight of potato and tomato caused by a complex of large-spored Alternaria species in Algeria. Pp. 167-179 in Schepers H.T.A.M. (ed.): Proceedings of the Sixteenth EuroBlight Workshop, 14-17 May 2017, Aarhus, Denmark.

Azouaoui-Idjer G, Della Rocca C, Pecchioli A, Bouznad Z, Danti R 2012. First report of Botryosphaeria iberica associated with dieback and tree mortality of Monterey Cypress (Cupressus macrocarpa) in Algeria. Plant Diseases, 96(7):1073, DOI: 10.1094/PDIS-10-110901-PDN. 
Battandier JA, Maire R, Trabut LC 1914. Rapport sur les herborisations faites par la société pendant la session d'Alger. Bulletin de la Société Botanique de France, 61 : 37-106.

Beddiar A, Adouane M, Boudiaf I, Fraga A 2015. Mycorrhizal status of main spontaneous or introduced forest trees in El Tarf province (Algerian North-east). The Online Journal of Science and Technology, 5 (2): 40-45.

Bekhouche F, Breton A, Gaillard-Martinie B 1994. Champignons cellulolytiques du sol des zones arides du Sahara algérien - mise en évidence de l'activité cellulasique. Cryptogamie-Mycologie, 15(2): 141-147.

Belhoucine L, Bouhraoua RT, Harrak MJ, Samson RA 2012. Les champignons associés à Platypus cylindrus (Coleoptera, Platypodidae) dans une subéraie du nord ouest d'Algérie: cas des champignons nuisibles. IOBC/wprs Bulletin, 76: 109-116 109.

Benbelkacem K, Bouznad Z, Benslimane H, Salhi NL 2011. Occurrence of septoria leaf blotch in Algéria and assessment of wheat resistance. International symposium on Mycrosphaerella and Stagnospora diseases of cereals, Mexico City, 10-14 September 2011.

Benia F, Pujade-Villar J 2014. Premiers signalements de Pseudocercospora neriella et Pseudomonas savastanoi nerii sur Nerium oleander L. en Algérie. Orsis 28: 153-159.

Benichou SL 2011. Etude de la variabilité génétique et phénotypique des champignons du genre Alternaria pathogènes des Apiacées en vue d'améliorer les méthodes de lutte. Doctorate Thesis, University Ahmed Ben Bella (Oran, Algeria), 170 pages.

Bensaci OA, Harzallah D, Gouaref K 2015. Endophytic mycoflora of Cedrus atlantica: diversity patterns and determinism of the phytosanitary situation of Atlas cedar forests in Belezma massif (Algeria). Forest Science and Technology, 11(1): 36-43, DOI: 10.1080/21580103.2014.957352.

Benslimane H, Lamari L, Benbelkacem A, Sayoud R, Bouznad Z 2011. Distribution of races of Pyrenophora tritici-repentis in Algeria and identication of a new virulence type. Phytopathol. Mediterr., 50: 203-211.

Benzeghmane A 2011. Inventaire des champignons isolés de palmes du palmier dattier (Phonix dactylifera) de quelques palmeraies de la région de Ouargla. Engineer Thesis, University Kasdi Merbah (Ouargla, Algeria), 83 pages.

Berlese AN 1890-1905. Icones Fungorum omnium hucusque cognitorum ad usum Sylloges Saccardianae adcommodatae, 5 volumes.
Berraf-Tebbal A, Guereiro MA, Phillips AJL 2014. Phylogeny of Neofusicoccum species associated with grapevine trunk diseases in Algeria, with description of Neofusicoccum algeriense sp. nov. Phytopathologia Mediterranea, 53(3): 416-427. DOI: 10.14601/Phytopathol_Mediterr-14385.

Bessadat N, Benichou S, Kihal M, Henni DE 2014. Aggressiveness and morphological variability of small spore Alternaria species isolated from Algeria. Journal of Experimental Biology and Agricultural Sciences, 2(2S): 265-278.

Bessadat N, Berruyer R, Hamon B, BatailleSimoneau N, Benichou S, Kihal M, Henni DE, Simoneau P 2017. Alternaria species associated with early blight epidemics on tomato and other Solanaceae crops in northwestern Algeria. Eur. J. Plant Pathol., 148(1): 181197, DOI 10.1007/s10658-016-1081-9.

Bessadat N, Hamon B, Henni DE, Simoneau P 2016. First report of tomato early blight caused by Alternaria grandis in Algeria. Plant Disease, 100(2): 533, DOI: 10.1094/PDIS-05-15-0613-PDN.

Boivin LH 1840. Enumération des plantes qui croissent spontanément en Barbarie. Revue Scientifique et Industrielle 3: 153-184.

Bordjiba O, Bekhouche F, Steiman R 2009. Biodegradation capability of some species of fungi isolated from contamined soils towards herbicides. Toxicology Letters, 189(Suppl.): S189.

Bouharati S, Harzallah D, Feni M, Benmahmed K 2008. The impact of fungi on Cedrus atlantica trees in the area around Al-Aures, Algeria: Environmental scanning data using artificial intelligence techniques. 13ème Congrès de l'Association Africaine pour la Fixation Biologique de l'Azote, Hammamet (Tunisia), 15-18 Décembre 2008, pp. 74.

Bouznad Z, Roquebert MF, Benelmouffok A 1995. Didymella viciae sp.nov., teleomorph of Ascochyta viciae Lib., pathogen of Vicia sativa in Algeria. Mycotaxon, 56: 443-450.

Braun U, Crous PW, Nakashima C 2014. Cercosporoid fungi (Mycosphaerellaceae). 2. Species on monocots (Acoraceae to Xyridaceae, excluding Poaceae). IMA Fungus, 5: 203-390.

Braun U, Crous PW, Nakashima C 2015. Cercosporoid fungi (Mycosphaerellaceae) 4. species on dicots (Acanthaceae to Amaranthaceae). IMA Fungus, 6(2): 373-469, DOI: 10.5598/imafungus.2015.06.02.09.

Breton A, Faurel L 1964. Deux espèces remarquables inédites de champignons coprophiles appartenant au genre Sporormia de Not. (sensu lato). Bull. 
Trimestriel de la Société Mycologique de France, 80(2): 247-258.

Chafai D 1996. Micromycètes des sédiments d'oueds et d'effluents industriels de l'Est algérien. Doctorate Thesis, University of Greboble (France), 145 pages.

Chamekh R, Deniel F, Donot C, Jany J-L, Nodet P, Belabid L 2019. Isolation, Identification and enzymatic activity of halotolerant and halophilic fungi from the Great Sebkha of Oran in northwestern of Algeria, Mycobiology, 47(2): 230-241, DOI: 10.1080/12298093.2019.1623979.

Chliyeh M, Touati J, Selmaoui K, Ouazzani TA, Filali MA., El Modafar CC, Moukhli A, Benkirane R, Allal DA 2014. Bibliographic inventory of the olive tree (Olea europaea L.) fungal diseases in the world. International Journal of Pure and Applied Bioscience, 2 (3): 46-79.

CMI (1954). Index of Fungi; Vol. 1; 1940-1949. Commonwealth Mycological Institute, Kew, United Kingdom, 430 pages.

CMI (1963). Index of Fungi; Vol. 2; 1950-1960. Commonwealth Mycological Institute, Institute, Kew, United Kingdom, 828 pages.

CMI (1968). Index of Fungi; Vol. 3; 1961-1966. Commonwealth Mycological Institute, Kew, United Kingdom, 376 pages.

CMI 1969. Index of fungi: a supplement to Petrak's lists, 1920-1939. Commonwealth Mycological Institute, Kew, 236 pages.

CMI (1982). Index of Fungi; Vol. 4; 1971-1980. Commonwealth Mycological Institute, Kew, United Kingdom, 643 pages.

Crous PW, Knox-Davies PS, Wingfield MJ1989. A List of eucalyptus leaf fungi and their potential importance to South African forestry. South African Forestry Journal, 149(1): 17-29, DOI: 10.1080/00382167.1989.9628988.

Debeaux O, Trabut A, Therry J, Combe AJ, Roumeguère $\mathrm{C} 1880$. Bouquet de champignons nouveaux observés dans le Midi de la France et en Algérie (18791880). Revue Mycologique 2(8): 187-191.

Dorleans G, Emmanouilidis I 1972. Présence d'un Coprin dans la Flore d'un chancre de néflier. Annales de l'Institut National Agronomique (Alger), 7: 21-25.

Dubost D 1966. Les Champignons des sols salés de l'Ouest Algérien. (1. Caractères généraux). Bulletin de la Société d'Histoire Naturelle de 1'Afrique du Nord, 57(12): 9-29.
Duby JÉ 1862. Mémoire sur la tribu des Hystérinées de la famille des Hypoxylées (Pyrénomycètes). Mémoires de la Société de Physique et d'Histoire Naturelle de Genève 16(1): 15-70.

Durieu MS 1846. Exploration Scientifique de l'Algérie. Paris: Imprimerie Impériale, 600 pages.

Faurel L., Schotter G. 1956. Un champignon parasite du géranium rosat en Algérie, Lasiodiplodia pezaliana n.sp. Bulletin de la Société d'Histoire Naturelle de l'Afrique du Nord 47 (3-4): 115-117, 3 figs.

Faurel L, Schotter G 1964. Notes mycologiques. II. Quelques champignons coprophiles des environs d'Alger. Revue de Mycologie Paris, N.S. 29 (4): 267-283.

Faurel L, Schotter G 1965. Notes Mycologiques IV. - Champignons coprophiles du Sahara central et notamment de la Tefedest. Revue de Mycologie, 30(3): 141-165.

Feldmann J 1932. Sur la répartition, dans la Mediterranée occidentale, du Melanopsamma tregoubovii Ollivier var. cystoseirae Oll. Pyrénomycète parasite du Cystoseira abroianifolia C. Ag. Revue Algologique, 6(2): 225-226.

Feldmann J 1944. Sur une Méliolacée (Ascomycètes, Erysiphales) vivant associée à une Cyanophycée. Bulletin de la Société d'Histoire Naturelle de l'Afrique du Nord, 35: 15-16.

Fröhlich J 1997. Biodiversity of microfungi associated with palms in the tropics. PhD Thesis, University of Hong Kong, 327 pages, DOI: 10.5353/th_b3123685.

Gibson IAS 1975. Diseases of forest trees widely planted as exotics in the tropics and Southern Hemisphere. Part I. The Myrtaceae. Commonwealth Forestry Inst. Kew, 63 pages.

Guechi A, Girre L 1994. Sources of Cycloconium oleaginum (Cast.) conidia for infection of olive leaves and conditions determining leaf spot disease development in the region of Sétif, Algeria. Mycopathologia, 125: 163171.

Harzallah D., Benssaci O.A., Bouharati S. (2009) Endophytic mycoflora of atlas cedar (Cedrus atlantica Man.) in Belezma massif (Aures, Algeria). Proceedings de la 9ème conférence internationale sur les maladies des plantes, Tours (France), 8-98 ET 9 December 2009, 10 pages.

Hennings PC 1901. Aliquot fungi Africae borealis a cl. Dr. G. Schweinfurth collecti. Hedwigia Beiblatt, 40 (4): $98-101$. 
Hennings PC 1902. Fungi Oranenses Hochreutinerani. In: Hochreutiner, B.P.G., Le SudOranais. Études floristiques et phytogéographiques faites au cours d'une exploration dans le sud-ouest de l'Algérie en 1901. Annuaire du Conservatoire et du Jardin Botaniques de Genève, 6:242-243.

Hyde KD, Jones EBG, Liu JK, Ariyawansa $\mathrm{H}$ et al. 2013 - Families of Dothideomycetes. Fungal Diversity $63,1-313$

Jaczewski ALA 1893. Quelques champignons récoltés en Algérie. Bulletin de la Société mycologique de France, 9: 46-51.

Jayasiri SC, Hyde KD, Jones EBG, McKenzie EHC, Jeewon R, Phillips AJL, Bhat DJ, Wanasinghe DN, Liu JK, Lu YZ, Kang JC, Xu J, Karunarathna SC (2019). Diversity, morphology and molecular phylogeny of Dothideomycetes on decaying wild seed pods and fruits. Mycosphere (10): 1-186.

Kachour L 2004. Identification des moisissures isolées à partir des eaux du lac Oubeira (PNEK) et impact des eaux usées sur leur diversité. Master Thesis, Université Badji Mokhtar (Annaba, Algeria), 260 pages.

Kahoul M, Amel Alioua A, Derbal N, Ayad W. 2014. Comportement des micromycètes du sol vis-à-vis de la pollution mercurielle dans la région d'Azzaba. J. Mater. Environ. Sci., 5 (5): 1470-147.

Kedad A, Bouznad Z 2018. Catalogue des champignons d'Algérie. Tarzalt M. Arts graphiques Edition, Alger - Algeria, 334 pages.

Kirk P M 1985. Saccardo's Omissions. [Index of Fungi Supplement.] Commonwealth Mycological Institute, Kew, 101 pages.

Kirk PM, Ansell AE 1992. Authors of fungal names. A list of authors of scientific names of fungi with recommended standard forms of their names, including abbreviations. Index of Fungi Supplement. CAB International, Kew, Surrey, Great Britain.

Kirk PM, Cannon PF, Minter DW, Stalpers JA 2008. Ainsworth \& Bisby's Dictionary of the Fungi, 10th ed. Wallingford, UK: CAB International.

Kohlmeyer J 1963. Répartition de champignons marins (Ascomycètes et Fungi Imperfecti) dans la Méditerranée. Rapports et procés-verbaux des réunions de la Commission internationale pour l'exploration scientifique de la Mer Méditerranée, 17: 723-730.

Lacoste L 1957. Champignons parasites et saprophytes de l'alfa (Stipa tenacissima L.). Revue de Mycologie. (Sup. Colon), 22: 6-18.
Ladjal S 2012. Activité antimicrobienne des métabolites secondaires des champignons endophytes isolés du pin d'Alep (Pinus halepensis Mill.) de la région de M'sila. Master Thesis, University Ferhat Abbas (Sétif, Algeria), 84 pages.

Lakhdari W 2017. Diagnosis of phytopathogenic fungi of Lycopersicon esculentum L. in Oued Righ region (Algerian Sahara). SDRP Journal of Plant Science, 1(1): 22-27.

Larignon P 2016. Maladies cryptogamiques du bois de la vigne: symptomatologie et agents pathogènes. http://www.vignevin.com, 168 pages.

Linaldeddu BT, Deidda A, Scanu B, Franceschini A, Serra A, Berraf-Tebbal A, Zouaoui Boutiti M, Ben Jamâa ML, Phillips AJL 2014. Diversity of Botryosphaeriaceae species associated with grapevine and other woody hosts in Italy, Algeria and Tunisia, with descriptions of Lasiodiplodia exigua and Lasiodiplodia mediterranea sp. nov. Fungal Diversity, 71: 201-214, DOI 10.1007/s13225-014-0301-x.

Linaldeddu BT, Franceschini A, Alves A, Phillips AJL 2013. Diplodia quercivora sp. nov.: a new species of Diplodia found on declining Quercus canariensis trees in Tunisia. Mycologia, 105(5): 1266-1274, DOI: 10.3852/12-370.

Locquin-Linard M 1977. Chaetopreussia chadefaudii n.g., n. sp. Revue de Mycologie, 41(2): 181187.

Locquin-Linard M 1988. Etude de la mycoflore coprophile des zones arides et semi-arides du nord de l'Afrique. Doctorate thesis, Université Paris VI (France), 244 pages.

Lunghini D 1978. Primo contributo alla conoscenza di alcuni ifali demaziacei del Sahara algerino, Giornale botanico italiano, 112(5-6): 373-393, DOI: 10.1080/11263507809427948.

Magnani G 1964. Diseases of Eucalyptus. Diseases of Widely Planted Forest Trees. FAO/IUFRO Symposium on Internationally Dangerous Forest Diseases and Insects. 159-167.

Maire R 1906. Contributions à l'étude de la flore mycologique de l'Afrique du Nord, Bulletin de la Société Botanique de France, 53: CLXXX-CCXV. DOI:10.1080/00378941.1906.10831941.

Maire R 1913. Études mycologiques. Annales Mycologici, 11(4) 351-358.

Maire R 1914. Deuxième contribution à l'étude de la flore mycologique de la Tunisie. Bulletin de la Société d'Histoire Naturelle de l'Afrique du Nord, 5: 254-260. 
Maire R. 1916 Schedae ad Mycothecam BorealiAfricanam. Ser. 2, fascs 8-9. Bulletin de la Société d'Histoire Naturelle de l'Afrique du Nord, 8: 294-303.

Maire R. 1917a. Champignons nord-africains nouveaux ou peu connus. Bulletin de la Société d'Histoire Naturelle de l'Afrique du Nord, 8: 131-200.

Maire R. 1917b. Schedae ad mycothecam borealiAfricanam. Bulletin de la Société d'Histoire Naturelle de 1'Afrique de Nord, 8(4): 74-84, 242-261.

Maire R. 1919. Schedae ad mycothecam borealiAfricanam. Bulletin de la Société d'Histoire Naturelle de l'Afrique de Nord, 10: 130-151.

Maire R. 1933. Mission du Hoggar II - Études sur la flore et la végétation du Sahara Central. Imprimerie la Typo-Litho, Alger, 271 pages.

Maire R. 1934. Sur quelques Champignons algériens. Bulletin de la Société d'Histoire Naturelle de l'Afrique du Nord, 25(1-2): 8-9, 39.

Maire R. 1945. Etudes mycologiques - Fascicule 5. Bulletin de la Société d'Histoire Naturelle de l'Afrique du Nord, 36(3): 24-42.

Maire R., Werner R.G. 1937. Fungi Maroccani. Mémoires de la Société des Sciences Naturelles du Maroc, 45: 1-147.

Maire, R. 1906. Notes Mycologiques. Annales Mycologici, 4(4): 329-335.

Manamgoda DS, Rossman AY, Castlebury LA, Crous PW, Madrid H, Chukeatirote E, Hyde KD 2014. The genus Bipolaris. Studies in Mycology, 79: 221-288, DOI: 10.1016/j.simyco.2014.10.002.

Mariat F, Liautaud B, Liautaud M, Marill FG 1978. Hendersonula toruloidea, agent d'une dermatite verruqueuse mycosique observée en Algérie. Sabouraudia, 16: 133-140.

Maublanc A 1903. Sur quelques espèces nouvelles de champignons inférieurs. Bull. Trimestriel Soc. Mycol. France 19: 291-296.

Montagne C 1856. Sylloge generum specierumque plantarum cryptogamarum. J.P. Baillière, Paris, 114 pages.

Montagne C 1857. Huitième centurie de plantes cellulaires nouvelles, tant indigènes qu'exotiques, Décades VI et VII. Annales des Sciences Naturelles, 8: 285-310.

Mouchacca J, Saint-Jore V 2000. Contribution of André Maublanc to mycology. II - taxonomic status of introduced taxa. Mycotaxon, 74(2): 301-330.
Nafady NA, Abdel-Azeem AM, Salem FM 2016. A checklist of Egyptian fungi: II. Glomeromycota. Microbial Biosystems Journal 1 (1): 40-49.

Ndiritu GG, Winsett KE, Spiegel FW, Stephenson SL 2009. A checklist of African myxomycetes. Mycotaxon, 107: 353-356.

Nezzar-Hocine H, Bouteville RJ, Halli-Hargas R, Chevalier G 1996. Fungal macroflora associated with Cedrus atlantica (Endl.) Manetti ex Carrière. I-Inventory in the the Djurjura mountains (Algeria). Cryptogamie Mycologie, 17(2): 85-103.

Nezzar-Hocine H, Bouteville RJ, Guimberteau J, Perrin R, Chevalier G 1998. Macrofungal flora associated with Cedrus atlantica (Endl.) Manetti ex Carrière II Ectomycorrhizal fungi in a cedar plantation (Djurjura mountains, Algeria). Cryptogamie - Mycologie, 19(1-2): 139-161.

Nicot J 1955. Remarques sur les peuplements de micromycètes des sables désertiques, C.R. Acad. Sci., 240: 2082-2084.

Nicot J 1955. Un apercu de la microflore fongique des sables désertiques. UNESCO Arid Zone Research, 5: 95-99.

Noumeur SR, Helaly SE, Jansen R, Gereke M, Stradal TEB, Harzallah D, Stadler M 2017. Preussilides A-F, Bicyclic Polyketides from the Endophytic Fungus Preussia similis with Antiproliferative Activity. Journal of natural products, 80(5):1531-1540.

Patouillard NT 1897a. Catalogue raisonné des plantes cellulaires de la Tunisie. Imprimerie Nationale, Paris, 158 pages.

Patouillard NT 1897b. Additions au catalogue des champignons de la Tunisie. Bulletin de la Société Mycologique de France, 13(4): 197-216.

Patouillard NT 1903. Additions au catalogue des champignons de la Tunisie. Bulletin de la Société Mycologique de France, 19(3): 254-261.

Patouillard NT (1904) Champignons algérotunisiens nouveaux ou peu connus, (Suite I). Bulletin de la Société Mycologique de France, 20: 51-54.

Petrak F. 1931. Mykologische Notizen XI. Annales Mycologici 29 (5-6): 339-397.

Pfister DH 1977. Annotated Index to Fungi Described by N. Patouillard. Contributions of Reed Herbarium, 25: 1-211.

Pons N, Sutton BC 1986. Cercospora and similar fungi on Heliotropium weeds. Mycol. Res., 100(7): 815820. 
Rebbouh S. 2016. Isolement et identification des champignons associés à la cochenille blanche Parlatoria blanchardi (Blanchard, 1868) sur quelques variétés de dattes. Master Thesis, University Kasdi Merbah (Ouargla - Algeria), 110 pages.

Roumeguère C 1882. Fungi Gallici exsiccati Centuria XXI. Revue Mycologique, 4(14): 96-105.

Roumeguère C 1883. Fungi Gallici exsiccatiCenturies XXIV-XXV. Revue Mycologique, 5(17): 6-30.

Roumeguère C 1885. Fungi exsiccati praecipue Gallici. Centurie XXXIV. Revue Mycologique, 7(26): 167-178.

Roumeguère C 1886.Fungi Gallici exsiccati (1) Centurie XXXIX. Revue Mycologique, 8(32): 190-200.

Roumeguère C 1887a. Fungi Gallici exsiccati Centurie XL. Revue Mycologique, 9(33): 19-29.

Roumeguère C 1887b. Fungi selecti exsiccati praecipue Galliae et Algeriae - Centurie XLI. Revue Mycologique, 9(34): 100-109.

Roumeguère C 1888a Fungi Europoei precipue Gallici exsiccati - Centurie XLIV. Revue Mycologique, 10(37): 8-17.

Roumeguère C 1888b. Fungi selecti exsiccati Centurie XLVII. Revue Mycologique, 10(40): 185-193.

Roumeguère C 1889. Fungi selecti exsiccati Centurie LI. Revue Mycologique, 11(44): 193-201.

Roumeguère C 1892. exsiccati precipue Gallici, LXème centurie. Revue Mycologique, 14(53): 1-11.

Saccardo PA 1881. Fungi Gallici lecti a cl. viris P. Brunaud, C.G. Gillet, Abb. Letendre, A. Malbranche, J. Therry vel editi in Mycotheca Gallica cl. C. Roumeguèri, recensuit P.A. Saccardo. Series III. Michelia, 2 (7): 302371.

Saccardo PA 1882. Sylloge fungorum omnium hucusque cognitorum - Volume I. Berolini: Fratres Borntraeger, Padua (Italy), 768 pages.

Saccardo PA 1883. Sylloge fungorum omnium hucusque cognitorum - Volume 2. Berolini: Fratres Borntraeger, Padua (Italy), 815 pages. .

Saccardo PA 1884. Sylloge fungorum omnium hucusque cognitorum - Volume 3. Berolini: Fratres Borntraeger, Padua (Italy), 860 pages.. .

Saccardo PA 1886. Sylloge fungorum omnium hucusque cognitorum - Volume 4. Berolini: Fratres Borntraeger, Padua (Italy), 807 pages. .

Saccardo PA 1891. Sylloge fungorum omnium hucusque cognitorum - Volume 9. Berolini: Fratres Borntraeger, Padua (Italy), 1141 pages. .
Saccardo PA 1931. Sylloge fungorum omnium hucusque cognitorum - Volume 25. Volume I. Berolini: Fratres Borntraeger, Padua (Italy), 1093 pages. .

Saccardo PA, Roumeguère C 1881. Fungi Algerienses Trabutiani - Sertulum II. Revue Mycologique, 3(9): 26-30.

Saccardo PA, Roumeguère C 1885. Fungi Algerienses, Tahitenses et Gallici. Revue Mycologique 7 (26): 158-161.

\section{Schneider-Orelli O (1913) 2. Einige} Beobachtungen Über die parasitischen Pilze Algeriens. Pp. In: Rikli M. \& Schröter C. (1912). Vom Mittelmeer zum Nordrand der Sahara - Eine botanische Frühlingsfahrt nach Algerien. - Zürich: Institut Orell Füssli. 166-170.

Schubert K, Ritschel A, Braun U 2003. A monograph of Fusicladium s.lat. (Hyphomycetes). Schlechtendalia, 9: 1-132.

Setti B, Bencheikh M, Henni J, Neema C 2009. Comparative aggressiveness of Mycosphaerella pinodes on peas from different regions in western Algeria. Phytopathologia Mediterranea, 48(2): 195-204.

Smahi H, Belhoucine-Guezouli L, Berraf-Tebbal A, Chouih S, Arkam M, Franceschini A, Linaldeddu BT, Phillips AJL 2017. Molecular characterization and pathogenicity of Diplodia corticola and other Botryosphaeriaceae species associated with canker and dieback of Quercus suber in Algeria. Mycosphere, 8(2): 1261-1272.

Söderström L, Hagborg A, von Konrat M, Renner MAM 2008. Early Land Plants Today: Liverwort checklist of checklists. Fieldiana, Botany, n.s. 47, 105130 .

Söderström L, Urmi E, Ván̆a J 2007. The distribution of Hepaticae and Anthocerotae in Europe and Macaronesia- Update 1-427. Cryptogamie, Bryologie 28, 299-350.

Stevenson JA 1926. Foreign Plant Diseases. A manual of economic plant diseases which are new to or not widely distributed in the United States. United States Department of Agriculture, Washington DC, USA. 196 pages.

Teponno RB, Noumeur SR, Helaly SE, Hüttel S, Harzallah D, Stadler M 2017. Furanones and Anthranilic Acid Derivatives from the Endophytic Fungus Dendrothyrium variisporum. Molecules; 22(10):16741686, DOI: 10.3390/molecules22101674. 
Trabut L 1891. Les champignons parasites du criquet pèlerin. Revue Générale de Botanique, 34: 401405.

Vouaux L 1912. Synopsis des champignons parasites de Lichens (Suite). - Bulletin de la Société Mycologique de France, 28: 177-208, 209 -256.

Winter G, Roumeguère C 1887. Champignons parasites des Eucalyptus. Revue Mycologique, 9 (33): 4142. 